\title{
Recursive weights for some Boolean functions
}

\author{
Alyssa Brown and Thomas W. Cusick \\ Communicated by Simon Blackburn
}

\begin{abstract}
This paper studies degree 3 Boolean functions in $n$ variables $x_{1}, \ldots, x_{n}$ which are rotation symmetric, that is, invariant under any cyclic shift of the indices of the variables. These rotation symmetric functions have been extensively studied in the last dozen years or so because of their importance in cryptography. Let $\operatorname{wt}(f)$ denote the (Hamming) weight of the Boolean function $f$. We extend and simplify results of Bileschi, Cusick and Padgett, who gave an algorithm for finding a recursion for the sequence $\left\{\operatorname{wt}\left(f_{r, s}^{n}\right): n=3,4, \ldots\right\}$, where $f_{r, s}^{n}=x_{1} x_{r} x_{s}+x_{2} x_{r+1} x_{s+1}+\cdots+x_{n} x_{r-1} x_{s-1}$ is the cubic rotation symmetric function generated by the monomial $x_{1} x_{r} x_{s}$. We show that the weights for the function $g_{r, s}^{n}=x_{1} x_{r} x_{s}+x_{2} x_{r+1} x_{s+1}+\cdots+x_{1+n-s} x_{r+n-s} x_{n}$ (note this is just the sum of the first $n-s+1$ terms in the definition of $f_{r, s}^{n}$ ) satisfy the same recursion as the weights for $f_{r, s}^{n}$. We prove the recursion for the weights of $g_{r, s}^{n}$ by a method based on the work by Bileschi-Cusick-Padgett, but we are able to avoid a lot of the complications in their proofs. In particular, we do not need the technical notion of " $g$ terms" or the elaborate proof of similarity of certain matrices.
\end{abstract}

Keywords. Boolean functions, rotation symmetry, Hamming weight, recursion.

2010 Mathematics Subject Classification. 94C10, 06E30, 94A60.

\section{Introduction}

The subject of Boolean functions is a well-known topic in cryptography and a detailed account of their applications can be found in [3]. A Boolean function $f(x)$ in $n$ variables is defined to be a map from the vector space $V_{n}$ in $n$ dimensions to the finite field $\mathrm{GF}(2)=\{0,1\}$.

Let the $2^{n}$ elements of $V_{n}$ be represented by $v_{0}=(0, \ldots, 0), v_{1}=(0, \ldots, 0,1)$, $\ldots, v_{2^{n}-1}=(1, \ldots, 1)$, where the vectors are listed in lexicographic order. We define the truth table of a Boolean function $f(x)$ to be the vector of length $2^{n}$ given by $\left(f\left(v_{0}\right), f\left(v_{1}\right), \ldots, f\left(v_{2^{n}-1}\right)\right)$. The truth table can also simply be written as a finite sequence $f\left(v_{0}\right) f\left(v_{1}\right) \ldots f\left(v_{2^{n}-1}\right)$. Furthermore, if we count the number of 1 's in the truth table, this gives the weight of the Boolean function.

Given a permutation $\rho$ on $n$ variables, defined such that $\rho\left(x_{1}, x_{2}, \ldots, x_{n}\right)=$ $\left(x_{2}, x_{3}, \ldots, x_{n}, x_{1}\right)$, let a Boolean function in $n$ variables, $f(x)$, where $x=$ 
$\left(x_{1}, x_{2}, \ldots, x_{n}\right)$, be called rotation symmetric if $f(x)=f(\rho(x))$ for all $x \in V_{n}$. A Boolean function is monomial rotation symmetric if it is rotation symmetric and generated by a single monomial. In [5] Piepryzyk and Qu demonstrated the desirability of rotation symmetric Boolean functions in cryptography by using them in fast hash functions. Since then, much attention has been given to rotation symmetric Boolean functions. In 2002, Cusick and Stănică [2] found a recursion for the weights of the monomial rotation symmetric Boolean functions generated by $x_{1} x_{2}$ and $x_{1} x_{2} x_{3}$ and gave a conjecture about the nonlinearities of the latter functions which was proved in 2010 by Zhang et al. [6]. Furthermore, in 2009, the weights and nonlinearity of quadratic monomial rotation symmetric Boolean functions were completely determined by Kim et al. in [4]. In 2011, Bileschi, Cusick and Padgett [1] gave an explicit algorithm for computing a recursion for the weights of any cubic monomial rotation symmetric function as the number of variables increases.

In this paper, we give another method for computing the recursions, first found in [1], for the weights of any cubic monomial rotation symmetric Boolean functions. To determine these weights, we begin by finding a recursion for the weights of simpler Boolean functions, also generated by a monomial, but where the permutation is not rotated and the function is cut short so that it will remain in $n$ variables. We then prove that the recursion for the weights of these two types of Boolean functions are the same. Furthermore, in determining the recursion of weights, we also determine a recursion for the truth tables of the two types of functions as well. Our method is based on the work in [1], but we are able to avoid many of the complications in the proofs in [1].

We use Xor or $\oplus$ to denote addition mod 2 of strings of bits. We use $a \| b$ to denote the juxtaposition of two strings $a, b$.

\section{Preliminaries}

Definition 1. Let $b^{n}$ denote the Boolean function $b$ in $n$ variables. Let $(1, r, s)_{n}=$ $f_{r, s}^{n}=x_{1} x_{r} x_{s}+\cdots+x_{n} x_{r-1} x_{s-1}$ denote the rotation symmetric Boolean function generated by $x_{1} x_{r} x_{s}$ in $n$ variables. Let $[1, r, s]_{n}=g_{r, s}^{n}$ denote the Boolean function $g: V_{n} \rightarrow \mathrm{GF}(2)$ given by $g(x)=x_{1} x_{r} x_{s}+x_{2} x_{r+1} x_{s+1}+\cdots+$ $x_{1+n-s} x_{r+n-s} x_{n}(n-s+1$ terms $)$.

For the remainder of this paper assume that $r$ and $s$ are fixed. Given these two values we can determine information about the Boolean function $g_{r, s}^{n}$ and the Boolean function $f_{r, s}^{n}$ for any value for $n$ such that $n \geq s$. 
Lemma 1. Let the truth table of the monomial $x_{1} x_{r} x_{s}$ in $n$ variables be denoted by $T_{n}(1, r, s)$. Then this truth table is given by (here $a_{j}$ as usual denotes a string of $j$ consecutive symbols $a, a \in\{0,1\})$ :

$$
T_{n}(1, r, s)=0_{2^{n-1}}\left(0_{2^{n-r}}\left(0_{2^{n-s}} 1_{2^{n-s}}\right)_{2^{s-r-1}}\right)_{2^{r-2}} .
$$

Proof. This statement is derived from [2, Lemma 11].

Definition 2. We say that a monomial $x_{i} x_{j} x_{k}$ in $n$ variables has action on a truth table $T\left(b^{n}\right)$ if the truth table of $x_{i} x_{j} x_{k}$ contains at least one 1 . In this case the action itself is defined to be $T\left(b^{n}\right) \oplus T\left(x_{i} x_{j} x_{k}\right)$. In a similar way, we can say that a monomial has action (or has no action) on a portion of a truth table $T\left(b^{n}\right)$ if the same portion of the truth table of $x_{i} x_{i} x_{k}$ has at least one 1 (or consists of all 0 's).

Definition 3. The stretch of a sequence, $b_{1} b_{2} \cdots b_{2^{k}}$, to a length of $2^{m}$ is defined to be $b_{1} \cdots b_{1} b_{2} \cdots b_{2} \cdots b_{2^{k}} \cdots b_{2^{k}}$, where each $b_{i}$ is repeated $2^{m-k}$ times.

Definition 4. Define an $m$-action on a truth table of a Boolean function $b^{n-1}$ called $m_{v}^{n}$-action (or $m_{v}^{n}$ for short; to simplify the notation we do not explicitly indicate the dependence on the fixed values of $r$ and $s$ ), where $v \in \mathbb{Z}, 1 \leq v \leq s$ and $n>s$, as follows: begin by finding the sequence $T_{n}(1, r, s)$ and split that sequence into $2^{s-v+1}$ equally sized portions. Take the last portion only and stretch it to a length of $2^{n-1}$. Call the resulting sequence of length $2^{n-1}$ the $m$-sequence for the $m_{v}^{n}$ action. Finally, the $m_{v}^{n}$-action applied to a Boolean function $b^{n-1}$ is defined to be

$$
\left.\begin{array}{c}
m_{v}^{n} \\
T\left(b^{n-1}\right)
\end{array}\right) T\left(b^{n-1}\right) \text { Xor } m,
$$

where $T\left(b^{n-1}\right)$ is the truth table of $b^{n-1}$ and $m$ is the $m$-sequence for the $m_{v}^{n}$ action.

Lemma 2. For any m-action $m_{v}^{n}$ with $s<n$ and $1 \leq v \leq s$ we can explicitly determine what the $m$-sequence for that $m$-action will be:

$$
\begin{array}{lll}
\text { 1. } & m=1_{2^{n-1}} & v=1, \\
\text { 2. } & m=\left(0_{\left.2^{n-v} 1_{2^{n-v}}\right)_{2^{v-2}}} 2 \leq v \leq s-r+1,\right. \\
\text { 3. } & m=\left(0_{\left.2^{n+s-r-v}\left(0_{2^{n-v}} 1_{2^{n-v}}\right)_{2^{s-r-1}}\right)_{2^{v+r-s-2}}}\right. & s-r+2 \leq v \leq s .
\end{array}
$$

Proof. 1 . When $v=1$, by Definition 4 we begin by splitting $T_{n}(1, r, s)$ into $2^{s}$ equally sized portions. Since $T_{n}(1, r, s)$ has length $2^{n}$ this means that each portion will have length $2^{n-s}$. By equation (1), the last $2^{n-s}$ digits in $T_{n}(1, r, s)$ are a sequence of all 1 's. We then stretch it to a length of $2^{n-1}$. 
2. When $2 \leq v \leq s-r+1$, we begin by splitting $T_{n}(1, r, s)$ into $2^{s-v+1}$ portions, which means that each portion will have length $2^{n-s+v-1}$. Now looking at equation (1), we see that $0_{2^{n-s}} 1_{2^{n-s}}$ repeats at the end of the sequence $2^{s-r-1}$ times, each of these subsequences having length $2^{n-s+1}$. Since $v-2 \leq s-r-1$ we can look at the subsequence consisting of the final $2^{v-2}$ copies of $0_{2^{n-s}} 1_{2^{n-s}}$. This sequence has a length of exactly $2^{n-s+v-1}$ and since it is the end of $T_{n}(1, r, s)$ we will take $\left(0_{2^{n-s}} 1_{2^{n-s}}\right)_{2^{v-2}}$ and stretch it to a length of $2^{n-1}$. By Definition 3, this means that each bit will be repeated $2^{s-v}$ times resulting in the $m$-sequence $\left(0_{2^{n-v}} 1_{2^{n-v}}\right)_{2^{v-2}}$.

3. If $s-r+2 \leq v \leq s$, when we split $T_{n}(1, r, s)$ we again end up with portions of length $2^{n-s+v-1}$. Furthermore, from equation (1) we see that $T_{n}(1, r, s)$ ends by repeating the sequence $0_{2^{n-r}}\left(0_{2^{n-s}} 1_{2^{n-s}}\right)_{2^{s-r-1}}$ exactly $2^{r-2}$ times. Since $0 \leq v+r-s-2 \leq r-2$ (because $v \leq s$ ) we can look at the subsequence where $0_{2^{n-r}}\left(0_{2^{n-s}} 1_{2^{n-s}}\right)_{2^{s-r-1}}$ repeats $2^{v+r-s-2}$ times. This subsequence has length $2^{n-r+1} \times 2^{v+r-s-2}=2^{n-s+v-1}$. Therefore, this is exactly the portion of $T_{n}(1, r, s)$ that we are looking for and hence we need to stretch it to a length of $2^{n-1}$. By Definition 3, we repeat each bit $2^{s-v}$ times. The resulting $m$-sequence is $\left(0_{2^{n+s-r-v}}\left(0_{2^{n-v}} 1_{2^{n-v}}\right)_{2^{s-r-1}}\right)_{2^{v+r-s-2}}$.

\section{Truth table recursion for $\boldsymbol{g}_{r, s}^{\boldsymbol{n}}$}

Lemma 3. Given a Boolean function $g_{r, s}^{n-1}$ in $n-1$ variables, define a new function in $n$ variables by $\sigma\left(g_{r, s}^{n-1}\right)=x_{2} x_{r+1} x_{s+1}+x_{3} x_{r+2} x_{s+2} \cdots x_{1+n-s} x_{r+n-s} x_{n}$. Then $T\left(\sigma\left(g_{r, s}^{n-1}\right)\right)=T\left(g_{r, s}^{n-1}\right) \| T\left(g_{r, s}^{n-1}\right)$.

Proof. Since $x_{1}$ does not appear in any term in $\sigma\left(g_{r, s}^{n-1}\right)$, the first and second halves of $T\left(\sigma\left(g_{r, s}^{n-1}\right)\right)$ must be the same. Clearly the first half of the truth table for $\sigma\left(g_{r, s}^{n-1}\right)$ is the first half of the truth table for the function $g_{r, s}^{n}\left(0, x_{2}, \ldots, x_{n}\right)$. By putting $y_{i}=x_{i+1}, 1 \leq i \leq n-1$, we see that this first half is the same as the truth table of $g_{r, s}^{n-1}\left(y_{1}, \ldots, y_{n-1}\right)$. Since the first and second halves are the same, we have now determined $T\left(\sigma\left(g_{r, s}^{n-1}\right)\right)$.

Lemma 4. We can generate the truth table $T\left(g_{r, s}^{n}\right)(s \leq n-1)$ recursively from the Boolean function $g_{r, s}^{n-1}$ in $n-1$ variables by

$$
T\left(g_{r, s}^{n}\right)=T\left(g_{r, s}^{n-1}\right) \| T\left(g_{s, s}^{n}\right) .
$$

Proof. We begin by noticing that $g_{r, s}^{n}=x_{1} x_{r} x_{s}+\sigma\left(g_{r, s}^{n-1}\right)$ and therefore that

$$
T\left(g_{r, s}^{n}\right)=T\left(x_{1} x_{r} x_{s}\right) \oplus T\left(\sigma\left(g_{r, s}^{n-1}\right)\right)=T_{n}(1, r, s) \oplus T\left(\sigma\left(g_{r, s}^{n-1}\right)\right) .
$$


Furthermore, since by Lemma 3, $T\left(\sigma\left(g_{r, s}^{n-1}\right)\right)=T\left(g_{r, s}^{n-1}\right) \| T\left(g_{r, s}^{n-1}\right)$, we will break this up into the first half and second half of the truth table.

In the first half of the truth table, $x_{1} x_{r} x_{s}$ has no action since the first half of $T_{n}(1, r, s)$ is all 0 's. Therefore, the first half of $T\left(g_{r, s}^{n}\right)$ is simply $T\left(g_{r, s}^{n-1}\right)$.

In the second half of the truth table, we need to account for the action of $x_{1} x_{r} x_{s}$. To do so, look at the $m_{s}^{n}$-action on $g_{r, s}^{n-1}$. By definition, we begin by finding $T_{n}(1, r, s)$ and split it into $2^{s-s+1}=2$ equal pieces. We take only the last piece, which here is just the second half. This sequence has length $2^{n-1}$ already and so to stretch it, nothing needs to be done. Finally, we Xor it with $T\left(g_{r, s}^{n-1}\right)$. It is clear that this result is the second half of $T\left(g_{r, s}^{n}\right)$ since it is the second half of $T_{n}(1, r, s)$ Xor-ed with the second half of $T\left(\sigma\left(g_{r, s}^{n-1}\right)\right)$.

\section{Recursion of weights for $g_{r, s}^{n}$}

Before we can determine a recursion formula for the weight of $g_{r, s}^{n}$ we need some preliminary definitions and lemmas.

Definition 5. We can split an $m$-action, called an $m$-action split or splitting an $m$-action, by splitting the $m$-sequence of the $m$-action into two equal pieces. Call the first half of the $m$-sequence $m_{1}$ and the second half $m_{2}$. Then $m_{1}$ Xor-ed with the first half of the truth table is defined to be the first half of the $m$-action split. Similarly, $m_{2}$ Xor-ed with the second half of the truth table is defined to be the second half of the $m$-action split.

Lemma 5. For any $m$-action $m_{v}^{n}$ with $s<n$ and $1 \leq v \leq s$, we can split this $m$-action such that the first half is one m-action and the second half is some other $m$-action. Also, given a truth table, $T\left(b^{n-1}\right)$ of some Boolean function in $n-1$ variables, let $T^{1}\left(b^{n-1}\right)$ represent the first half of the truth table and $T^{2}\left(b^{n-1}\right)$ represent the second half. Then

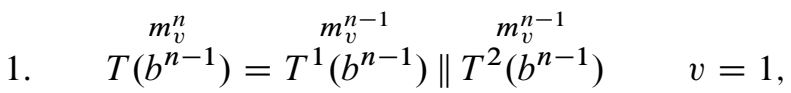

2. $\left.\quad \begin{array}{c}m_{v}^{n} \\ n\left(b^{n-1}\right)\end{array}\right)=T^{1}\left(b^{n-1}\right) \| T^{2}\left(b^{n-1}\right) \quad v=s-r+2, v=2$,

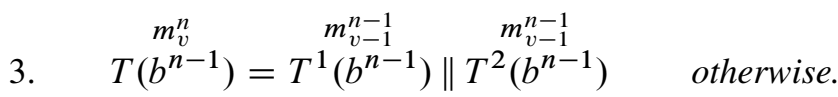

Proof. First note that to show the first half and the second half of an $m$-action are as defined above, we need only show that the first half and the second half 
of the $m$-sequence in the $m_{v}^{n}$-action match up with the $m$-sequence from the $m$ action on the first half and the $m$-action on the second half of the split $m_{v}^{n}$-action, respectively. In other words, the $m$-sequence resulting from the $m$-action on the left-hand side of the equation should equal the concatenation of the $m$-sequences resulting from the $m$-actions on the right-hand side.

Case 1: From Lemma 2, when $v=1$ the $m$-sequence from the $m_{1}^{n}$-action is a sequence of all 1's of length $2^{n-1}$. Similarly, the $m$-sequence resulting from the action $m_{1}^{n-1}$ is also a sequence of 1's, this time of length $2^{n-2}$. If we take this sequence and concatenate it with itself, then we simply have the $m$-sequence from $m_{1}^{n}$.

Case 2: If we have $v=2$ then the $m$-sequence resulting from the $m_{2}^{n}$-action is $0_{2^{n-2}} 1_{2^{n-2}}$ by Lemma 2 . The first half of the $m$-sequence is all zeros, so the first half of the truth table will be unchanged. Therefore, the first half of the $m$-action split should consist of no $m$-action. The second half is a sequence of all 1 's of length $2^{n-2}$. Therefore, the second half of the $m$-action split is $m_{1}^{n-1}=m_{v-1}^{n-1}$.

If we have $v=s-r+2$, then by Lemma 2 the $m$-sequence is $0_{2^{n+s-r-v}}\left(0_{2^{n-v}} 1_{2^{n-v}}\right)_{2^{s-r-1}}$. The first half of this $m$-sequence is all zeros and so the first half of the $m$-action split consists of no $m$-action. The second half of this $m$-sequence is clearly $\left(0_{2^{n-v}} 1_{2^{n-v}}\right)_{2^{s-r-1}}$ which can be rewritten as $\left(0_{2^{(n-1)-(v-1)}} 1_{\left.2^{(n-1)-(v-1)}\right)} 2_{2^{(v-1)-2}}\right.$. From Lemma 2 it is clear that this is just an $m_{v-1}^{n-1}$ action where $v-1=s-r+1$.

Case 3: If $2<v \leq s-r+1$ then the $m$-sequence is $\left(0_{2^{n-v}} 1_{2^{n-v}}\right)_{2^{v-2}}$ and if $s-r+2<v \leq s$ then the $m$-sequence is $\left(0_{2^{n+s-r-v}}\left(0_{2^{n-v}} 2_{2^{n-v}}\right)_{2^{s-r-1}}\right)_{2^{v+r-s-2}}$. In both of these cases the sequences consist of a portion that repeats an even number of times; therefore, it is clear that the first half of the $m$-sequence equals the second half of the $m$-sequence. Furthermore, in the former case, we can write the first (and second) half of the $m$-sequence as $\left(0_{2^{(n-1)-(v-1)}} 1_{2^{(n-1)-(v-1)}}\right)_{2^{(v-1)-2}}$, which is the $m$-sequence for $m_{v-1}^{n-1}$ when $2<v \leq s-r+1$. In the latter case, the first (and hence the second) half of the $m$-sequence can be written as $\left(0_{2^{(n-1)+s-r-(v-1)}}\left(0_{2^{(n-1)-(v-1)}} 1_{2^{(n-1)-(v-1)}}\right)_{2^{s-r-1}}\right)_{2^{(v-1)+r-s-2}}$, which is the $m$-sequence for $m_{v-1}^{n-1}$ when $s-r+2<v \leq s$.

Definition 6. For some fixed integers $n$ and $s<n$, the complete set of $m_{v}^{n}$-actions is $\left\{m_{1}^{n}, m_{2}^{n}, \ldots, m_{s}^{n}\right\}$. We can denote any subset of this set by $O_{j}^{n}$ where $0 \leq j<$ $2^{s}$. Namely, we write $j$ in binary and prepend it with zeros until it has length $s$. Then, $m_{i}^{n} \in O_{j}^{n}$ if and only if the $i$ th place of $j$ written in binary is a 1 (we count places from left to right and begin counting at $i=1$ ). For example, $O_{2^{s}-1}^{n}$ is the complete set because $2^{s}-1$ in binary is a sequence of length $s$ consisting of all 1's. Also, $O_{0}^{n}=\emptyset$ and $O_{1}^{n}=\left\{m_{s}^{n}\right\}$. We call any of these sets $O_{j}^{n}$ to be performed on a truth table an operation. 
Definition 7. We can now define an operation split, also called splitting an operation. Given an operation $O_{j}^{n}$ to be applied to the truth table of $g_{r, s}^{n-1}$ we can split this into a first half $O_{w_{1}}^{n-1}$ and a second half $O_{w_{2}}^{n-1}$ such that $O_{w_{1}}^{n-1}$ will be applied to the first half of the truth table and $O_{w_{2}}^{n-1}$ will be applied to the second half of the truth table. In particular, for all $m$-actions $m_{v}^{n} \in O_{j}^{n}$, use Lemma 5 to determine how the $m$-action splits. Put the first half of the $m$-action split into the first half of the operation split and the second half of the $m$-action split into the second half of the operation split. In addition, by Lemma 4 we must add the $m$-action $m_{s}^{n-1}$ to the second half of the operation split because we are also splitting the truth table of $g_{r, s}^{n-1}$. Use the complete list of $m$-actions in the first and second half of the operation split to determine the values of $w_{1}$ and $w_{2}$.

An algorithm for this process is explicitly defined below in Remark 1.

From Lemma 4 we know that

$$
T\left(g_{r, s}^{n}\right)=T\left(g_{r, s}^{n-1}\right) \| T\left(g_{r, s}^{n}\right) .
$$

Now, applying Definitions 6 and 7 we can rewrite this as

$$
\begin{array}{ccc}
O_{0}^{n+1} & O_{0}^{n} & O_{1}^{n} \\
T\left(g_{r, s}^{n}\right) & =T\left(g_{r, s}^{n-1}\right) \| & T\left(g_{r, s}^{n-1}\right) .
\end{array}
$$

Using Definition 7 again we could break down this equation further to get

$$
\begin{array}{ccccc}
O_{0}^{n+1} & O_{0}^{n-1} & O_{1}^{n-1} & O_{w_{1}}^{n-1} & O_{w_{2}}^{n-1} \\
T\left(g_{r, s}^{n}\right)= & T\left(g_{r, s}^{n-2}\right)\left\|T\left(g_{r, s}^{n-2}\right)\right\| T\left(g_{r, s}^{n-2}\right) \| T\left(g_{r, s}^{n-2}\right),
\end{array}
$$

where the values of $w_{1}$ and $w_{2}$ are determined by the process described in Definition 7. This process of expansion repeated $k$ times is called the $k$ th level of expansion. Notice that if for some expansion we have an $m$-action $m_{i}^{n}$ applied twice to the same portion of the truth table, then we can remove $m_{i}^{n}$ from the list of $m$-actions to be applied on that portion since applying it twice simply cancels it out. The examples at the end of the paper show how equations (2) and (3) work.

Using equations (2) and (3) above as a guide, we can see that in the $k$ th level of expansion we have $2^{k}$ copies of $T\left(g_{r, s}^{n-k}\right)$ concatenated together, each with some $O_{i}^{n-k+1}$ operation above it, $0 \leq i<2^{s}$.

Definition 8. Represent the $k$ th level of expansion as a column vector where the $i$ th place in the vector is the number of times $O_{i}^{n-k+1}$ appears in the expansion. Therefore, the vector has length $2^{s}$. Furthermore, recall from Lemma 2 that an $m_{1}^{n-k+1}$-action has an $m$-sequence which is all 1's. Therefore, this $m$-action 
simply complements the truth table of the Boolean function. If for some $j$ we have $m_{1}^{n-k+1} \in O_{j}^{n-k+1}$ (note this implies $j \geq 2^{s-1}$ ), then write $O_{j}^{n-k+1}=$ $\left\{m_{1}^{n-k+1}\right\} \cup O_{i}^{n-k+1}$ where $i=j-2^{s-1}$. Now if $O_{j}^{n-k+1}$ appears in the $k$ th level of expansion instead of representing it by adding a 1 to the $j$ th row, add a -1 to the $i$ th row. Also, to keep track of the number of times this event happens, we will add a single extra entry to the end of the column vector and add a 1 to it every time the event occurs. In this way, we can shorten the column vector to a length of $2^{s-1}+1$. Call this result the column vector of the kth level of expansion.

Definition 9. Similar to the definition above, we now define the column vector of the expansion of $O_{j}^{n}$, which will have length $2^{s-1}+1$. To construct this vector, begin by splitting the operation $O_{j}^{n}$ according to Definition 7. Let $O_{w_{1}}^{n-1}$ be the operation to be applied to the first half of the truth table and $O_{w_{2}}^{n-1}$ be the operation to be applied to the second half of the truth table. If $w_{1}<2^{s-1}$, then add a 1 to entry $w_{1}$ of the vector (begin counting with zero). If $w_{1} \geq 2^{s-1}$, then add a -1 to entry $w_{1}-2^{s-1}$ and a 1 to entry $2^{s-1}$, the last entry. Follow the same process for $w_{2}$. Let all other entries in the vector be zero.

The main difference between this vector and the column vector of the kth level of expansion is that the operation $O_{j}^{n}$ always splits into precisely two pieces, rather than the $k$ th level of expansion, which consists of $2^{k}$ pieces. So in the column vector of the expansion of $O_{j}^{n}$ there are always only two operations represented.

Now we define a $\left(2^{s-1}+1\right) \times\left(2^{s-1}+1\right)$ matrix $A$. The first $2^{s-1}$ columns of $A$ will be the column vector of the expansion of $O_{j}^{n}$ for $0 \leq j \leq 2^{s-1}-1$. The last column of the matrix $A$ is defined to be all zeros except for a 2 in the last entry. This column represents all of the column vectors of the expansion $O_{j}^{n}$ for $j \geq 2^{s-1}$. The first $2^{s-1}$ entries of this column are zero because the operations that appear in the expansion are represented by the entries in the previous columns. The last entry is a 2 because each of the $O_{j}^{n}$ represented in this column contains $m_{1}^{n}$, which splits to $m_{1}^{n-1}$ in the first and second half, by Lemma 5. It is helpful to look at Example 4 below to see how the matrix $A$ is constructed.

Lemma 6. $A^{k} \cdot v$ is the column vector of the $k$ th level of expansion, where $v=$ $\left(\begin{array}{llll}1 & 0 & \cdots & 0\end{array}\right)^{T}$.

Proof. To prove that $A^{k} \cdot v$ is the column vector of the $k$ th level of expansion, we will use induction. In the base case we have $k=1$. It is clear that the first column of $A$ is the column vector of the first level of expansion by the construction of $A$ and the fact that $O_{0}^{n}=\emptyset$. Now, by the induction hypothesis, assume that $A^{k-1} \cdot v$ is the column vector of the $(k-1)$ th level of expansion. Then the first column 
of $A^{k-1}$ represents a list of all operations that need to be split to determine the column vector of the $k$ th level of expansion. To calculate the first column of $A^{k}$, take the first column of $A^{k-1}$ and dot product it with each row of $A$. Every nonzero entry of the column represents an operation that needs to be split; let entry $j$ be represented by $c_{j}$. When we dot product the column with row $i$ of $A$, if element $a_{i j}$ is nonzero, then we know that $O_{i}^{n-k-1}$ appears in the split of the operation $O_{j}^{n-k}$, and so the dot product adds it to the $i$ th entry if $c_{j} \neq 0$. If the product $c_{j} \cdot a_{i j}$ is negative, that means we have the complement, and so the dot product will subtract it instead. This is consistent with what we want because applying an operation and the complement of that operation to a portion of a truth table results in taking the complement of that portion. Therefore, we remove both operations and instead we want to use the complement. This is the purpose of the last row of the matrix. We have kept track of the number of times a complement appears, and so applying the dot product on the last row of $A$ will add up the number of times the complement appears. Therefore, the first column of $A^{k}$ is as desired and by induction, $A^{k} \cdot v$ is the column vector of the $k$ th level of expansion.

The following lemma offers an algorithm for calculating the matrix $A$.

Lemma 7. We can redefine the matrix $A$ as the sum of two matrices $R_{1}$ and $R_{2}$, so that $R_{1}+R_{2}=A$. Precisely, we can define $R_{1}$ by: for $0 \leq i<2^{s-1}$, write $i$ in binary using $s$ bits. If the $(s-r+2)$ th place of $i$ in binary (counting from left to right and starting at 1) is $a 0$, then column $i$ has a 1 at entry $2 i$ or entry $2 i-2^{s-1}$ if the second entry of $i$ in binary is a 0 or 1 , respectively. If the $(s-r+2)$ th place of $i$ in binary is a 1 , then column $i$ has a 1 at entry $2 i-2^{r-1}$ or entry $2 i-2^{s-1}-2^{r-1}$ if the second entry of $i$ is a 0 or 1 , respectively. When $i=2^{s-1}$, there is $a 1$ in the last entry of the column. All other entries are zero. We can define $R_{2}$ by: for $0 \leq i<2^{s-2}$, column $i$ has a 1 at entry $2 i+1$. For $2^{s-2} \leq i<2^{s-1}$, column $i$ has -1 at entry $2 i+1-2^{s-1}$ and a 1 in the last entry. When $i=2^{s-1}$, there is $a 1$ in the last entry. All other entries are zero.

Proof. Intuitively, we know that the matrix $A$ keeps track of the splits of all operations. $R_{1}$ simply keeps track of only the left half of these splits and $R_{2}$ keeps track of the right half. Let us first look at $R_{1}$.

First recall from Lemma 4 and Definition 7 that the left half of an operation split never has a new $m$-action to add in and so we only need to worry about how the existing $m$-actions split. Also notice that $R_{1}$ will never have any negative entries since when an $m_{v}^{n}$-action splits, the left-hand side will contain $m_{1}^{n-1}$ if and only if $v=1$, by Lemma 5. Furthermore, $m_{1}^{n} \in O_{i}^{n}$ if and only if $i \geq 2^{s-1}$. These operations are all represented by the last column, so we put a 1 in the last entry of 
the last column to represent the $m_{1}^{n-1}$ contained in the left side of these operation splits. For all of the previous columns we have $0 \leq i \leq 2^{s-1}-1$. Let $i$ written in binary be $(i)_{2}=b_{1} b_{2} \cdots b_{s}$ (where we prepend with zeros to make $i$ have length $s$ if necessary) and let the left-hand side of the operation split of $O_{i}^{n}$ be $O_{w_{1}}^{n-1}$. If $b_{j}=0$ for some $j$ then $m_{j-1}^{n} \notin O_{w_{1}}^{n-1}$ (by Lemma 5) and so we want the $(j-1)$ th place of $w_{1}$ in binary to be zero. If $b_{j}=1$ for some $j$, then $m_{j}^{n} \in O_{i}^{n}$. If furthermore $j \neq s-r+2$ and $j \neq 2$, then we will have $m_{j-1}^{n-1} \in O_{w_{1}}^{n-1}$ by Lemma 5 and therefore the $(j-1)$ th place of $w_{1}$ will be a 1 . If $b_{2}=1$ then $m_{2}^{n} \in O_{j}^{n}$ and notice that the left side of this $m$-action split does not contain an $m$-action, so we want the first place of $w_{1}$ in binary to be a zero. If $b_{s-r+2}=1$ then similarly, $m_{s-r+2}^{n} \in O_{j}^{n}$ and the left side of this $m$-action split does not contain an $m$-action and so we want the $(s-r+1)$ th place of $w_{1}$ in binary to be a zero. In general, we want the $(j-1)$ th place of $w_{1}$ in binary to equal the $j$ th place of $i$ in binary, except for $j=2$ and $j=s-r+2$. In these two cases we always want the $(j-1)$ th place of $w_{1}$ to be zero.

Now notice that $(2 i)_{2}=b_{1} b_{2} \cdots b_{s} 0$. Also, since $i \leq 2^{s-1}-1$, we have $b_{1}=0$, and $(2 i)_{2}=b_{2} \cdots b_{s} 0$. If $b_{2}=0$ and $b_{s-r+2}=0$, then it is clear that we want $w_{1}=2 i$. If $b_{2}=1$ and $b_{s-r+2}=0$, then to ensure that the first place of $w_{1}$ is a 0 and not a 1 , let $w_{1}=2 i-2^{s-1}$. Similarly, if $b_{2}=0$ and $b_{s-r+2}=1$, let $w_{1}=2 i-2^{r-1}$ to ensure that the $(s-r+1)$ th place of $w_{1}$ is a 0 . Finally, if $b_{2}=1$ and $b_{s-r+2}=1$ let $w_{1}=2 i-2^{r-1}-2^{s-1}$.

Now look at the matrix $R_{2}$. Let $O_{w_{2}}^{n-1}$ be the right side of the operation split of $O_{i}^{n}$, or, if $m_{1}^{n-1}$ is contained in the right side, then let $O_{w_{2}}^{n-1} \cup\left\{m_{1}^{n-1}\right\}$ be the right side of the operation split. Using Definition 7 we can see that we will need to add $m_{s}^{n-1}$ to the list of $m$-actions that will be applied to the right half of the truth table. This means that the last, or sth place of $w_{2}$ will be a 1 for every column $i, 0 \leq i \leq 2^{s-1}-1$. Also, using a similar analysis as we did for $R_{1}$ we can see that whenever $m_{j}^{n} \in O_{i}^{n}$ then $m_{j-1}^{n-1} \in O_{w_{2}}^{n-1}$. Hence, we want the $(j-1)$ th place of $w_{2}$ in binary to equal the $j$ th place of $i$ in binary. Furthermore, one additional consideration is that when $i \geq 2^{s-2}$ we have that $m_{2}^{n} \in O_{i}^{n}$ and by Lemma $5, m_{1}^{n-1}$ is contained in the right side of the expansion, and so we instead use $O_{w_{2}}^{n-1} \cup\left\{m_{1}^{n-1}\right\}$ as the right side of the operation split. This means that instead of using a 1 in that column we will need -1 instead, along with a 1 in the last entry to keep track of this occurrence.

Now notice that if $(i)_{2}=b_{1} b_{2} \cdots b_{s}$, then $2 i+1=b_{2} b_{3} \cdots b_{s} 1$. If $0 \leq i \leq$ $2^{s-1}-1$, then this is exactly what we want for $w_{2}$, so let $w_{2}=2 i+1$. If $2^{s-1} \leq$ $i<2^{s}$, then to account for taking the complement, let $w_{2}=2 i+1-2^{s-1}$.

Remark 1. We can use Lemma 7 as an algorithm for determining how each operation splits. For $0 \leq j<2^{s-1}$, we can split $O_{j}^{n}$ into $O_{w_{1}}^{n-1}$ on the left and $O_{w_{2}}^{n-1}$ on 
the right. If the $(s-r+2)$ th place of $j$ in binary (counting from left to right and starting at 1) is a 0 , then $w_{1}=2 j$ or $w_{1}=2 j-2^{s-1}$ if the second place of $j$ is a 0 or a 1 , respectively. If the $(s-r+2)$ th place of $j$ is a 1 , then $w_{1}=2 j-2^{r-1}$ or $w_{1}=2 j-2^{s-1}-2^{r-1}$ if the second place of $j$ is a 0 or a 1 , respectively. For any value of $j, 0 \leq j<2^{s-1}$, we have $w_{2}=2 j+1$. The proof of this is the same as the proof for Lemma 7 except that the need for taking complements is eliminated.

Furthermore, we can extend this to include the remaining possible values of $j$, $2^{s-1} \leq j<2^{s}$. When $j$ is in this upper range,

$$
O_{j}^{n}=O_{j-2^{s-1}}^{n} \cup\left\{m_{1}^{n}\right\} .
$$

Therefore, we can split $O_{j}^{n}$ by the above rules, except using $j-2^{s-1}$ instead of $j$. Additionally, we need to then take the complement of the left and right side of the operation split. To do this, for the values of $w_{1}$ and $w_{2}$ found, use $w_{1} \oplus 2^{s-1}$ and $w_{2} \oplus 2^{s-1}$ instead (we regard $w_{1}, w_{2}$, and $2^{s-1}$ as being written in binary).

We can now find a relationship between the matrix $A$ and the weight of the Boolean function $g_{r, s}^{n}$.

Lemma 8. The following equation holds:

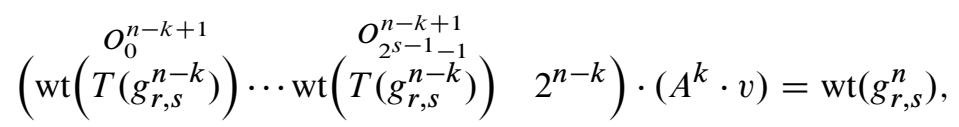

where $v=\left(\begin{array}{llll}1 & 0 & \cdots & 0\end{array}\right)^{T}$.

Proof. $A^{k} \cdot v$ is the column vector of the $k$ th level of expansion by Lemma 6 and so entry $i$ of $A^{k} \cdot v$ is the coefficient of the number of times $O_{i}^{n-k+1}$ is performed on $T\left(g_{r, s}^{n-k}\right)$. A negative coefficient means that the complement of $O_{i}^{n-k+1}$ is performed and the weight of a complement is

$$
2^{n-k}-\operatorname{wt}\left(T\left(g_{r, s}^{n-k+1}\right)\right)
$$

Also recall that the $k$ th level of expansion of a truth table is the concatenation of $2^{k}$ copies of $T\left(g_{r, s}^{n-k}\right)$ and each copy has some operation applied to it (even if it is just the identity operation, $O_{0}^{n-k+1}$ ). Therefore, every copy is counted in the column vector and the weight of the function is simply the sum of the weights of the portions being concatenated together. If $A^{k} \cdot v=\left(\begin{array}{lll}c_{1} & c_{2} \cdots c_{2^{s-1}+1}\end{array}\right)^{T}$, then

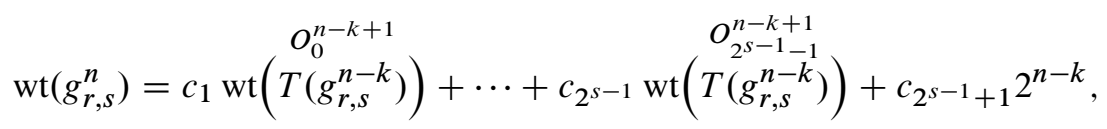

which is simply the equation above. 
Theorem 1. Let $m(x)=x^{k}+d_{k-1} x^{k-1}+\cdots+d_{1} x+d_{0}$ be the minimal polynomial of the matrix $A$ defined above. Then

$$
\operatorname{wt}\left(g_{r, s}^{n}\right)=-d_{k-1} \operatorname{wt}\left(g_{r, s}^{n-1}\right)-\cdots-d_{1} \operatorname{wt}\left(g_{r, s}^{n-(k-1)}\right)-d_{0} \operatorname{wt}\left(g_{r, s}^{n-k}\right) .
$$

Proof. By the Cayley-Hamilton theorem we have that

$$
A^{k}+d_{k-1} A^{k-1}+\cdots+d_{1} A+d_{0} I=0 .
$$

Furthermore, we can dot product on the right by $v=\left(\begin{array}{llll}1 & 0 & \cdots & 0\end{array}\right)^{T}$ and on the left by

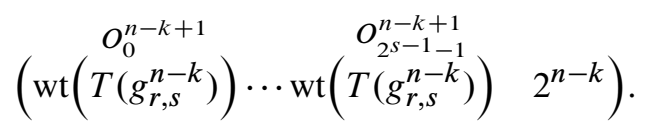

Then, distributing through the polynomial, we have that term $i$ is

$$
\begin{aligned}
& \left(\begin{array}{c}
O_{0}^{n-k+1} \\
\operatorname{wt}\left(T\left(g_{r, s}^{n-k}\right)\right)
\end{array}\right) \cdots \mathrm{wt}\left(\begin{array}{c}
O_{2^{s-1}-1}^{n-k+1}\left(g_{r, s}^{n-k}\right) \\
\left(g^{n-k}\right.
\end{array}\right) \cdot A^{k-i} \cdot v
\end{aligned}
$$

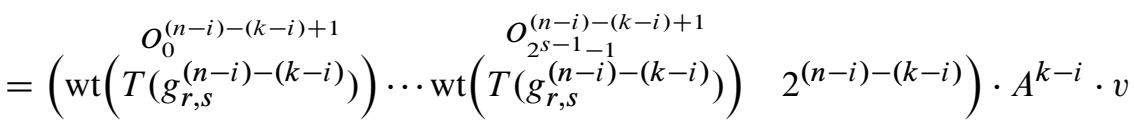

$$
\begin{aligned}
& =\operatorname{wt}\left(g_{r, s}^{n-i}\right)
\end{aligned}
$$

by Lemma 8 . Using this process on each term we get

$$
\mathrm{wt}\left(g_{r, s}^{n}\right)+d_{k-1} \operatorname{wt}\left(g_{r, s}^{n-1}\right)+\cdots+d_{1} \operatorname{wt}\left(g_{r, s}^{n-(k-1)}\right)+d_{0} \mathrm{wt}\left(g_{r, s}^{n-k}\right)=0 .
$$

\section{Truth table recursion for $\boldsymbol{f}_{\boldsymbol{r}, \boldsymbol{s}}^{\boldsymbol{n}}$}

In the next section we will give a recursion for the weights of monomial rotation symmetric Boolean functions $f_{r, s}^{n}$, in terms of the recursion for the weights of the Boolean functions $g_{r, s}^{n}$ already found in Theorem 1. In this section we find a recursion for the truth tables of the functions $f_{r, s}^{n}$ and we will use this as a tool in the following section. Begin by noticing that

$$
\begin{aligned}
f_{r, s}^{n}= & x_{1} x_{r} x_{s}+\cdots+x_{n-s+1} x_{n-s+r} x_{n}+x_{n-s+2} x_{n-s+r+1} x_{1}+\cdots \\
& \quad+x_{n-r+1} x_{n} x_{s-r}+x_{n-r+2} x_{1} x_{s-r+1}+\cdots+x_{n} x_{r-1} x_{s-1} \\
= & g_{r, s}^{n}+g_{r^{\prime}, s^{\prime}}^{n}+g_{r^{\prime \prime}, s^{\prime \prime}}^{n},
\end{aligned}
$$

where $r^{\prime}=n-s+2, s^{\prime}=n-s+r+1, r^{\prime \prime}=s-r+1$, and $s^{\prime \prime}=n-r+2$. Note that $g_{r^{\prime}, s^{\prime}}^{n}$ has $s-r$ monomials and $g_{r^{\prime \prime}, s^{\prime \prime}}^{n}$ has $r-1$ monomials. Also note 
here that the values of $r^{\prime}, s^{\prime}$, and $s^{\prime \prime}$ are dependent on $n$. So $g_{r^{\prime}, s^{\prime}}^{n}$ and $g_{r^{\prime \prime}, s^{\prime \prime}}^{n}$ will have different subscripts than $g_{r^{\prime}, s^{\prime}}^{n-1}$ and $g_{r^{\prime \prime}, s^{\prime \prime}}^{n-1}$ because the value of $n$ changes.

To find the truth table of $f_{r, s}^{n}$ we will use the truth table for $g_{r, s}^{n}$ as a base and then Xor it with the truth table for each monomial in $g_{r^{\prime}, s^{\prime}}^{n}$, and $g_{r^{\prime \prime} s,{ }^{\prime \prime}}^{n}$. A recursion for the truth table of $f_{r, s}^{n}$ will be done the same way. We already know the truth table recursion for $g_{r, s}^{n}$ by equation (2). To determine the truth table recursions for the monomials in $g_{r^{\prime}, s^{\prime}}^{n}$ and $g_{r^{\prime \prime}, s^{\prime \prime}}^{n}$ we will need some preliminary definitions and lemmas before we can begin.

Definition 10. Partition a truth table, $T\left(b^{n}\right)$, into $2^{a}$ equally sized portions. Then $T_{a}^{k}\left(b^{n}\right)$ represents the $k$ th portion of this divided table, for $1 \leq k \leq 2^{a}$.

The following is derived from [2, Lemma 11, p. 298]:

Lemma 9. The truth table of $x_{i} x_{j} x_{k}$ in $n$ variables where $1 \leq i<j<k \leq n$ is

$$
\left(0_{2^{n-i}}\left(0_{2^{n-j}}\left(0_{2^{n-k}} 1_{2^{n-k}}\right)_{2^{k-j-1}}\right)_{2^{j-i-1}}\right)_{2^{i-1}} .
$$

First let us look at the monomials included in $g_{r^{\prime}, s^{\prime}}^{n}$. Let

$$
h_{i}^{n}=x_{i} x_{n-s+1+i} x_{n-s+r+i} \quad \text { for } i=1, \ldots, s-r .
$$

Then $g_{r^{\prime}, s^{\prime}}^{n}$ is the sum of these $s-r$ monomials $h_{i}^{n}$.

Lemma 10. The truth table recursion for $h_{i}^{n}, 1 \leq i \leq s-r$, is given by

$$
\begin{aligned}
T\left(h_{i}^{n}\right)= & \left(T_{i}^{1}\left(h_{i}^{n-1}\right)\left\|T_{i}^{1}\left(h_{i}^{n-1}\right)\right\| T_{i}^{2}\left(h_{i}^{n-1}\right) \| T_{i}^{2}\left(h_{i}^{n-1}\right)\right)_{2^{i-1}} \\
= & T_{i}^{1}\left(h_{i}^{n-1}\right)\left\|T_{i}^{1}\left(h_{i}^{n-1}\right)\right\| T_{i}^{2}\left(h_{i}^{n-1}\right)\left\|T_{i}^{2}\left(h_{i}^{n-1}\right)\right\| \\
& \cdots\left\|T_{i}^{2^{i}}\left(h_{i}^{n-1}\right)\right\| T_{i}^{2^{i}}\left(h_{i}^{n-1}\right) .
\end{aligned}
$$

We think of this split as being the concatenation of $2^{i}$ pairs, for a total of $2^{i+1}$ pieces.

Remark 2. Although the two equations from Lemma 10 appear different, they do both give the same recursion. Use of the former equation decreases computation when actually computing such a truth table via the recursion and also gives more insight into the pattern of the recursion. The latter equation is more general and would require more computation, but in fact, using this equation when determining a recursion for the weights will greatly simplify those computations, as we will see in the following section. 
Proof. First note that by Lemma 9 we have

$$
T\left(h_{i}^{n}\right)=\left(0_{2^{n-i}}\left(0_{2^{s-i-1}}\left(0_{2^{s-r-i}} 1_{2^{s-r-i}}\right)_{2^{r-2}}\right)_{2^{n-s}}\right)_{2^{i-1}}
$$

and

$$
T\left(h_{i}^{n-1}\right)=\left(0_{2^{n-i-1}}\left(0_{2^{s-i-1}}\left(0_{2^{s-r-i}} 1_{2^{s-r-i}}\right)_{2^{r-2}}\right)_{2^{n-s-1}}\right)_{2^{i-1}} .
$$

Now split $T\left(h_{i}^{n-1}\right)$ into $2^{i-1}$ pieces. Each piece is identical and we begin to isolate some portions of it:

$$
\underbrace{0_{2^{n-i-1}}}_{T_{i}^{1}\left(h_{i}^{n-1}\right)} \underbrace{\left(0_{2^{s-i-1}}\left(0_{2^{s-r-i}} 1_{2^{s-r-i}}\right)_{2^{r-2}}\right)_{2^{n-s-1}}}_{T_{i}^{2}\left(h_{i}^{n-1}\right)},
$$

where the subscript $i$ on each $T$ is used because by taking $2^{i-1}$ portions and then splitting them each in half, we have split the table in $2^{i}$ pieces. Also notice that due to the repetition of $T\left(h_{i}^{n-1}\right)$ the first half of each of these $2^{i-1}$ portions is $T_{i}^{1}\left(h_{i}^{n-1}\right)$ and the second half is $T_{i}^{2}\left(h_{i}^{n-1}\right)$.

Now notice that when we split $T\left(h_{i}^{n}\right)$ into $2^{i-1}$ portions the first half of each portion becomes simply $T_{i}^{1}\left(h_{i}^{n-1}\right) \| T_{i}^{1}\left(h_{i}^{n-1}\right)$ and the second half similarly becomes $T_{i}^{2}\left(h_{i}^{n-1}\right) \| T_{i}^{2}\left(h_{i}^{n-1}\right)$. Therefore, we have proven the first of the two equations in the lemma. To see that the second equation is equivalent, note that equation (4) will repeat $2^{i-1}$ times in $T\left(h_{i}^{n-1}\right)$. Each time it repeats, instead of fixing the superscripts on each $T$ to be 1 and 2, allow them to increase with respect to the location in the table. Then, for each of these portions, "doubling up" the first and the second halves will give the equivalent portion on $T\left(h_{i}^{n}\right)$. This proves the second equation in the lemma.

Remark 3. Notice further that by repeating this process we could break $T\left(h_{i}^{n}\right)$, for each $1 \leq i \leq s-r$, into $2^{k}$ pieces where $i+1 \leq k \leq n-s+i$ :

$$
T\left(h_{i}^{n}\right)=\left(\left(T_{i}^{1}\left(h_{i}^{n-k+i}\right)\right)_{2^{k-i}} \|\left(T_{i}^{2}\left(h_{i}^{n-k+i}\right)\right)_{2^{k-i}}\right)_{2^{i-1}}
$$

or, equivalently

$$
\begin{aligned}
& T\left(h_{i}^{n}\right) \\
& =\underbrace{T_{i}^{1}\left(h_{i}^{n-k+i}\right)\|\cdots\| T_{i}^{1}\left(h_{i}^{n-k+i}\right)}_{2^{k-i} \text { times }}\|\cdots\| \underbrace{T_{i}^{2^{i}}\left(h_{i}^{n-k+i}\right)\|\cdots\| T_{i}^{2^{i}}\left(h_{i}^{n-k+i}\right)}_{2^{k-i} \text { times }},
\end{aligned}
$$

where we have $k \leq n-s+i$ because we must have the number of variables of the function, $n-k+i$, greater than or equal to $s$. Also note that by choosing $n$ large enough we can have large values of $k$. 
We now turn our attention to $g_{r^{\prime \prime}, s^{\prime \prime}}^{n}$. Let $\tilde{h}_{i}^{n}=x_{i} x_{s-r+i} x_{n-r+1+i}$ for $i=$ $1, \ldots, r-1$. Then $g_{r^{\prime \prime}, s^{\prime \prime}}^{n}$ is the sum of these $r-1$ monomials $\tilde{h}_{i}^{n}$.

Lemma 11. The truth table recursion for $\tilde{h}_{i}^{n}, 1 \leq i \leq r-1$, is given by

$$
\begin{aligned}
T\left(\tilde{h}_{i}^{n}\right)=( & \left(\left(T_{s-r+i}^{1}\left(\tilde{h}_{i}^{n-1}\right)\right)_{2}\right)_{2^{s-r}} \| \\
& \left.\left(\left(T_{s-r+i}^{2^{s-r}+1}\left(\tilde{h}_{i}^{n-1}\right)\right)_{2} \|\left(T_{s-r+i}^{2^{s-r}+2}\left(\tilde{h}_{i}^{n-1}\right)\right)_{2}\right)_{2^{s-r-1}}\right)_{2^{i-1}}
\end{aligned}
$$

or, equivalently

$$
T\left(\tilde{h}_{i}^{n}\right)=T_{s-r+i}^{1}\left(\tilde{h}_{i}^{n-1}\right)\left\|T_{s-r+i}^{1}\left(\tilde{h}_{i}^{n-1}\right)\right\| \cdots\left\|T_{s-r+i}^{2^{s-r+i}}\left(\tilde{h}_{i}^{n-1}\right)\right\| T_{s-r+i}^{2^{s-r+i}}\left(\tilde{h}_{i}^{n-1}\right)
$$

a split consisting of $2^{s-r+i}$ pairs, or $2^{s-r+i+1}$ pieces.

Proof. First note that by Lemma 9 we have that

$$
T\left(\tilde{h}_{i}^{n}\right)=\left(0_{2^{n-i}}\left(0_{2^{n-s+r-i}}\left(0_{2^{r-i-1}} 1_{2^{r-i-1}}\right) 2^{n-s}\right) 2_{2^{s-r-1}}\right)_{2^{i-1}}
$$

and

$$
T\left(\tilde{h}_{i}^{n-1}\right)=\left(0_{2^{n-i-1}}\left(0_{2^{n-s+r-i-1}}\left(0_{2^{r-i-1}} 1_{2^{r-i-1}}\right)_{2^{n-s-1}}\right)_{2^{s-r-1}}\right)_{2^{i-1}} .
$$

Now split $T\left(\tilde{h}_{i}^{n-1}\right)$ into $2^{i-1}$ pieces. Each piece is identical, but in order to match it with $T\left(\tilde{h}_{i}^{n}\right)$ we will need to break it up further, another $2^{s-r+1}$ times:

$$
\begin{aligned}
0_{2^{n-i-1}}\left(0_{2^{n-s+r-i-1}}\left(0_{2^{r-i-1}} 1_{2^{r-i-1}}\right)_{2^{n-s-1}}\right)_{2^{s-r-1}} & =(\underbrace{0_{2^{n-i-s+r-1}}}_{T_{s-r+i}^{1}\left(\tilde{h}_{i}^{n-1}\right)})^{s-r}(\underbrace{0_{2^{n-s+r-i-1}}^{\left(0_{2}\right.}}_{T_{s-r+i}^{2^{s-r}+1}\left(\tilde{h}_{i}^{n-1}\right)} \underbrace{\left.0_{1} 1_{2^{r-i-1}}\right)_{2^{n-s-1}}}_{T_{s-r+i}^{2^{s-r}+2}\left(\tilde{h}_{i}^{n-1}\right)})_{2^{s-r-1}} .
\end{aligned}
$$

This gives a total of $2^{i-1} \times 2^{s-r+1}=2^{s-r+i}$ equally sized portions, and hence, the subscript $s-r+i$ is used on each $T$ above. Also, due to the repetition inside $T\left(\tilde{h}_{i}^{n-1}\right)$, the superscripts on each $T$ can remain the same for each repetition of a portion.

Finally, notice that by "doubling up" each of the bracketed portions above in equation (5), we get the first equation for $T\left(\tilde{h}_{i}^{n}\right)$ given in the lemma. To see the equivalency of the second equation, instead of allowing the superscripts of each $T$ to remain fixed when a portion is repeated, instead increase the superscripts accordingly. Then "double up" each of these portions. This will give the second equation from the lemma. 
Remark 4. Also, similar to the previous remark, we could repeat this process to break $T\left(\tilde{h}_{i}^{n}\right)$, for each $1 \leq i \leq r-1$, into $2^{k}$ portions with $s-r+i+1 \leq k \leq$ $n-r+i$ :

$$
T\left(\tilde{h}_{i}^{n}\right)=\left(A_{2^{s-r}} \| B_{2^{s-r-1}}\right)_{2^{i-1}},
$$

where

$$
\begin{aligned}
& A=\left(T_{s-r+i}^{1}\left(\tilde{h}_{i}^{n-k-r+s+1}\right)\right)_{2^{k-s+r-i}}, \\
& B=\left(T_{s-r+i}^{2^{s-r}+1}\left(\tilde{h}_{i}^{n-k-r+s+1}\right)\right)_{2^{k-s+r-i}} \|\left(T_{s-r+i}^{2^{s-r}+2}\left(\tilde{h}_{i}^{n-k-r+s+1}\right)\right)_{2^{k-s+r-i}}
\end{aligned}
$$

or, equivalently

$$
\begin{aligned}
T\left(\tilde{h}_{i}^{n}\right)=\underbrace{T_{s-r+i}^{1}\left(\tilde{h}_{i}^{n-k-r+s+i}\right)\|\cdots\| T_{s-r+i}^{1}\left(\tilde{h}_{i}^{n-k-r+s+i}\right)}_{2^{k-s+r-i} \text { times }} \| \\
\cdots \| \underbrace{T_{s-r+i}^{2^{s-r+i}}\left(\tilde{h}_{i}^{n-k-r+s+i}\right)\|\cdots\| T_{s-r+i}^{2^{s-r+i}}\left(\tilde{h}_{i}^{n-k-r+s+i}\right)}_{2^{k-s+r-i} \text { times }} .
\end{aligned}
$$

Notice that for every monomial in $g_{r^{\prime}, s^{\prime}}^{n}$ and $g_{r^{\prime \prime}, s^{\prime \prime}}^{n}$ the first recursion for the truth table splits the table into exactly $2^{i+1}, 1 \leq i \leq s-r$, and $2^{s-r+i+1}$, $1 \leq i \leq r-1$, pieces, respectively. Therefore, the maximum number of splits one could get from doing each of these recursions is $2^{s}$.

To make the recursion of the truth table for $f_{r, s}^{n}$ uniform, we would like to take the recursion of the truth table for $g_{r, s}^{n}$ and all the remaining monomials and write them as tables split into $2^{s}$ portions. Namely, by using equation (3) and the remarks following it, we can determine an equation for the $s$ th level of expansion for $g_{r, s}^{n}$ :

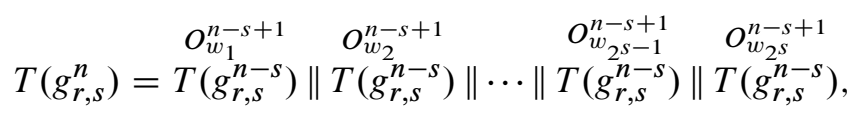

where the operations $O_{w_{j}}^{n-s+1}$ are determined by repeatedly using Definition 7 .

Furthermore, by Remark 3, for each $1 \leq i \leq s-r$, we can split $T\left(h_{i}^{n}\right)$ into $2^{s}$ pieces:

$$
\begin{aligned}
T\left(h_{i}^{n}\right) & =\left(\left(T_{i}^{1}\left(h_{i}^{n-s+i}\right)\right)_{2^{s-i}} \|\left(T_{i}^{2}\left(h_{i}^{n-s+i}\right)\right)_{2^{s-i}}\right)_{2^{i-1}} \\
& =\underbrace{T_{i}^{1}\left(h_{i}^{n-s+i}\right)\|\cdots\| T_{i}^{1}\left(h_{i}^{n-s+i}\right)}_{2^{s-i} \text { times }}\|\cdots\| \underbrace{T_{i}^{2^{i}}\left(h_{i}^{n-s+i}\right)\|\cdots\| T_{i}^{2^{i}}\left(h_{i}^{n-s+i}\right)}_{2^{s-i} \text { times }}
\end{aligned}
$$


and by Remark 4 , for each $1 \leq i \leq r-1$, we can split $T\left(\tilde{h}_{i}^{n}\right)$ into $2^{s}$ pieces:

$$
\begin{aligned}
T\left(\tilde{h}_{i}^{n}\right)= & \left(\left(T_{s-r+i}^{1}\left(\tilde{h}_{i}^{n-r+i}\right)\right)_{2^{r-i}}\right)_{2^{s-r}} \| \\
& \left.\left(\left(T_{s-r+i}^{2^{s-r}+1}\left(\tilde{h}_{i}^{n-r+i}\right)\right)_{2^{r-i}} \|\left(T_{s-r+i}^{2^{s-r}+2}\left(\tilde{h}_{i}^{n-r+i}\right)\right)_{2^{r-i}}\right)_{2^{s-r-1}}\right)_{2^{i-1}} \\
= & \underbrace{T_{s-r+i}^{1}\left(\tilde{h}_{i}^{n-r+i}\right)\|\cdots\| T_{s-r+i}^{1}\left(\tilde{h}_{i}^{n-r+i}\right) \|}_{2^{r-i} \text { times }} \\
& \cdots \| \underbrace{T_{s-r+i}^{2^{s-r+i}}\left(\tilde{h}_{i}^{n-r+i}\right)\|\cdots\| T_{s-r+i}^{2^{s-r+i}}\left(\tilde{h}_{i}^{n-r+i}\right)}_{2^{r-i} \text { times }} .
\end{aligned}
$$

Definition 11. We say that two equally sized portions of a truth table have the same structure if the sequences making up the two portions are identical, except for some operations that are applied to each portion (the operations on each portion may be different).

For example, the first half and the second half of $T\left(g_{r, s}^{n}\right)$ have the same structure, as can be seen in equation (2).

Theorem 2. The truth table recursion for $f_{r, s}^{n}$ is given by $T\left(f_{r, s}^{n}\right)=$

$$
T\left(g_{r, s}^{n-s}\right) \bigoplus_{i=1}^{s-r} T_{s}^{1}\left(h_{i}^{n}\right) \bigoplus_{i=1}^{O_{w_{1}}^{n-s+1}} T_{s}^{1}\left(\tilde{h}_{i}^{n}\right)\|\cdots\| T\left(g_{r, s}^{n-s}\right) \bigoplus_{i=1}^{s-r} T_{s}^{2^{s}}\left(h_{i}^{n}\right) \bigoplus_{i=1}^{r-1} T_{s}^{2^{s}}\left(\tilde{h}_{i}^{n}\right) .
$$

By looking at equations (7) and (8) we can easily determine $T_{s}^{j}\left(h_{i}^{n}\right)$ and $T_{s}^{j}\left(\tilde{h}_{i}^{n}\right)$.

Furthermore, if we take the $2^{s}$ portions of $T\left(f_{r, s}^{n}\right)$ and pair them up from left to right, for each pair of portions, $(j, j+1)$ (where $j$ is an odd integer and $\left.1 \leq j<2^{s}\right)$, then

$$
T\left(g_{r, s}^{n-s}\right) \bigoplus_{i=1}^{s-r} T_{s}^{j}\left(h_{i}^{n}\right) \bigoplus_{i=1}^{r-1} T_{s}^{j}\left(\tilde{h}_{i}^{n}\right)=T\left(g_{r, s}^{n-s}\right) \bigoplus_{i=1}^{s-r} T_{s}^{j+1}\left(h_{i}^{n}\right) \bigoplus_{i=1}^{r-1} T_{s}^{j+1}\left(\tilde{h}_{i}^{n}\right) .
$$

In particular, the elements of each pair have the same structure.

Proof. Split $T\left(f_{r, s}^{n}\right)$ into $2^{s}$ equally sized portions and look at each portion individually. It is clear from equation (6) and the earlier work in this section that the $j$ th portion of $T\left(f_{r, s}^{n}\right)$ is

$$
T\left(g_{r, s}^{n-s}\right) \bigoplus_{i=1}^{n-s+1} T_{s}^{j}\left(h_{i}^{n}\right) \bigoplus_{i=1}^{r-1} T_{s}^{j}\left(\tilde{h}_{i}^{n}\right)
$$


Furthermore, since performing the operation $O_{w_{j}}^{n-s+1}$ simply Xor's a sequence onto $T\left(g_{r, s}^{n-s}\right)$ it does not matter if we do that first and then Xor on the monomials or Xor the monomials onto $T\left(g_{r, s}^{n-s}\right)$ first and then perform the operation. Then by concatenating each of these portions together we get the first equation in the lemma.

To see that each pair has the same structure, we need only show that for $j$ an odd integer and $1 \leq j<2^{s}, T_{s}^{j}\left(h_{i}^{n}\right)=T_{s}^{j+1}\left(h_{i}^{n}\right)$ for all $i=1, \ldots, s-r$ and $T_{s}^{j}\left(\tilde{h}_{i}^{n}\right)=T_{s}^{j+1}\left(\tilde{h}_{i}^{n}\right)$ for all $i=1, \ldots, r-1$.

By equation (7), for all $i=1, \ldots, s-r$, we have $T_{s}^{j}\left(h_{i}^{n}\right)=T_{i}^{j^{\prime}}\left(h_{i}^{n-s+i}\right)$ for some $j^{\prime}$. Since each $T_{i}^{j^{\prime}}\left(h_{i}^{n-s+i}\right)$ is repeated an even number of times and $j$ is an odd integer, we must also have that $T_{s}^{j+1}\left(h_{i}^{n}\right)=T_{i}^{j^{\prime}}\left(h_{i}^{n-s+i}\right)$.

By equation (8), for all $i=1, \ldots, r-1$, we have $T_{s}^{j}\left(\tilde{h}_{i}^{n}\right)=T_{s-r+i}^{j^{\prime}}\left(\tilde{h}_{i}^{n-s+r}\right)$ for some $j^{\prime}$. Since each $T_{s-r+i}^{j^{\prime}}\left(\tilde{h}_{i}^{n-s+r}\right)$ is repeated an even number of times and $j$ is an odd integer, we must also have that $T_{s}^{j+1}\left(\tilde{h}_{i}^{n}\right)=T_{s-r+i}^{j^{\prime}}\left(\tilde{h}_{i}^{n-s+r}\right)$.

Remark 5. From this point on a new notation will be used for the recursion of $T\left(f_{r, s}^{n}\right)$. Let the $j$ th portion identified above,

$$
T\left(g_{r, s}^{n-s}\right) \bigoplus_{i=1}^{s-r} T_{s}^{j}\left(h_{i}^{n}\right) \bigoplus_{i=1}^{O_{w_{j}}^{n-s+1}} T_{s}^{j}\left(\tilde{h}_{i}^{n}\right),
$$

be represented by $T_{s}^{j}$. We choose a subscript $s$ since the table is split into a total of $2^{s}$ pieces. Also, by Theorem $2, T_{s}^{j}=T_{s}^{j+1}$ when $j$ is an odd number. Therefore, we can rewrite the recursion of $T\left(f_{r, s}^{n}\right)$ using this simpler notation:

$$
T\left(f_{r, s}^{n}\right)=\begin{array}{cccccccccc}
O_{w_{1}}^{n-s+1} & O_{w_{2}}^{n-s+1} & O_{w_{3}}^{n-s+1} & O_{w_{4}}^{n-s+1} & & O_{w_{2} s+1}^{n-s+1} & O_{w_{2} s}^{n-s+1} \\
T_{s}^{1} & T_{s}^{1} & \| & T_{s}^{3} & \| & T_{s}^{3} & \|\cdots\| T_{s}^{2^{s}-1} & \| T_{s}^{2^{s}-1}
\end{array} .
$$

Also notice here that if we wish to split the table further into more pieces, then $T_{s}^{j}=T_{s+1}^{j} \| T_{s+1}^{j}$. We could repeat this process $k$ times to get

$$
T_{s}^{j}=\underbrace{T_{s+k}^{j}\|\cdots\| T_{s+k}^{j}}_{2^{k} \text { times }},
$$

where

$$
T_{s+k}^{j}=T\left(g_{r, s}^{n-s-k}\right) \bigoplus_{i=1}^{s-r} T_{s+k}^{j}\left(h_{i}^{n}\right) \bigoplus_{i=1}^{r-1} T_{s+k}^{j}\left(\tilde{h}_{i}^{n}\right) .
$$




\section{Recursion of weights for $f_{r, s}^{n}$}

Definition 12. We say a recursion for weights for a truth table $T\left(b^{n}\right)$, where $b^{n}$ is a Boolean function in $n$ variables, is induced by a polynomial $m(x)$ if the first half of the table, say

$$
\begin{gathered}
O_{w_{1}}^{n} \\
T\left(b^{n-1}\right),
\end{gathered}
$$

and the second half, say

$$
\begin{gathered}
O_{w_{2}}^{n} \\
T\left(b^{n-1}\right),
\end{gathered}
$$

have the same structure, and there exists a matrix $A$ such that

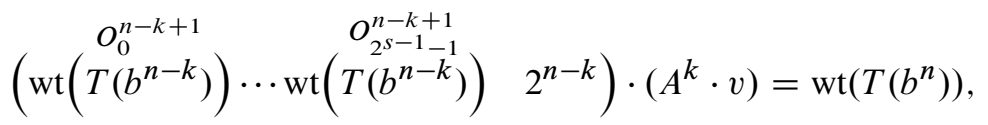

where $v$ is some initial vector and $m(x)$ is the minimal polynomial of $A$.

For example, a recursion of weights for $T\left(g_{r, s}^{n}\right)$ is induced by a polynomial as seen in Lemma 8. Furthermore, once given the minimal polynomial $m(x)=$ $x^{k}+d_{k-1} x^{k-1}+\cdots+d_{1} x+d_{0}$, it defines a recursion for the weights of the truth table by using the fact that $m(A)=0$ to get

$\operatorname{wt}\left(T\left(b^{n}\right)\right)=-d_{k-1} \operatorname{wt}\left(T\left(b^{n-1}\right)\right)-\cdots-d_{1} \operatorname{wt}\left(T\left(b^{n-(k-1)}\right)\right)-d_{0} \operatorname{wt}\left(T\left(b^{n-k}\right)\right)$.

Lemma 12. Let a given truth table be divided into two equally sized portions. If the first half and the second half of the table both satisfy a recursion of the weights defined by the same polynomial $m(x)$, then the whole truth table satisfies the recursion of weights defined by the polynomial $m(x)$.

Proof. Let $T\left(b^{n}\right)$ be a truth table of a Boolean function in $n$ variables and let $T^{1}\left(b^{n}\right)$ and $T^{2}\left(b^{n}\right)$ be the first half and the second half of the table, respectively. Notice that $\operatorname{wt}\left(T\left(b^{n}\right)\right)=\operatorname{wt}\left(T^{1}\left(b^{n}\right)\right)+\operatorname{wt}\left(T^{2}\left(b^{n}\right)\right)$.

Let recursions for weights of $T^{1}\left(b^{n}\right)$ and $T^{2}\left(b^{n}\right)$ both be defined by $m(x)=$ $x^{k}+d_{k-1} x^{k-1}+\cdots+d_{1} x+d_{0}$. Then we have

$$
\begin{aligned}
\operatorname{wt}\left(T^{1}\left(b^{n}\right)\right)=- & d_{k-1} \operatorname{wt}\left(T^{1}\left(b^{n-1}\right)\right)-\cdots-d_{1} \operatorname{wt}\left(T^{1}\left(b^{n-k-1}\right)\right) \\
& \quad-d_{0} \operatorname{wt}\left(T^{1}\left(b^{n-k}\right)\right), \\
\operatorname{wt}\left(T^{2}\left(b^{n}\right)\right)=- & d_{k-1} \operatorname{wt}\left(T^{2}\left(b^{n-1}\right)\right)-\cdots-d_{1} \operatorname{wt}\left(T^{2}\left(b^{n-k-1}\right)\right) \\
& \quad-d_{0} \operatorname{wt}\left(T^{2}\left(b^{n-k}\right)\right) .
\end{aligned}
$$


This implies that

$$
\begin{aligned}
\operatorname{wt}\left(T^{1}\left(b^{n}\right)\right)+\operatorname{wt}\left(T^{2}\left(b^{n}\right)\right)=- & d_{k-1}\left(\operatorname{wt}\left(T^{1}\left(b^{n-1}\right)\right)+\operatorname{wt}\left(T^{2}\left(b^{n-1}\right)\right)\right) \\
& -\cdots-d_{0}\left(\operatorname{wt}\left(T^{1}\left(b^{n-k}\right)\right)+\operatorname{wt}\left(T^{2}\left(b^{n-k}\right)\right)\right)
\end{aligned}
$$

or

$\operatorname{wt}\left(T\left(b^{n}\right)\right)=-d_{k-1} \operatorname{wt}\left(T\left(b^{n-1}\right)\right)-\cdots-d_{1} \operatorname{wt}\left(T\left(b^{n-k-1}\right)\right)-d_{0} \operatorname{wt}\left(T\left(b^{n-k}\right)\right)$.

Therefore, if the first half and the second half of a truth table satisfy the same recursion for weights, then the whole table satisfies that recursion for weights as well.

Lemma 13. For each pair of splits in the truth table recursion for $T\left(f_{r, s}^{n}\right)$, say

$$
\begin{aligned}
& O_{w_{j}}^{n-s+1} \quad O_{w_{j+1}}^{n-s+1} \\
& T_{s}^{j} \| T_{s}^{j},
\end{aligned}
$$

as given in Remark 5, the matrix A defined in Section 4 also satisfies

$$
\begin{aligned}
\left(\operatorname{wt}\left(\begin{array}{c}
O_{0}^{n-s-k+1} \\
T_{s+k}^{j}
\end{array}\right) \cdots \operatorname{wt}\left(\begin{array}{c}
O_{2^{s-1}-1}^{n-s-k+1} \\
T_{s+k}^{j}
\end{array}\right) 2^{n-s-k}\right) \cdot\left(A^{k} \cdot v\right) & \\
& =\operatorname{wt}\left(\begin{array}{ccc}
O_{w_{j}}^{n-s+1} & O_{w_{j}+1}^{n-s+1} \\
T_{s}^{j} & \| & T_{s}^{j}
\end{array}\right)
\end{aligned}
$$

where $v$ is the zero vector of length $2^{s-1}+1$ and we add 1 to place $w_{j}$ if $w_{j}<$ $2^{s-1}$, or we add -1 to place $w_{j}-2^{s-1}$ and 1 to place $2^{s-1}$ if $w_{j} \geq 2^{s-1}$; similarly, we also add 1 to place $w_{j+1}$ if $w_{j+1}<2^{s-1}$, or we add -1 to place $w_{j+1}-2^{s-1}$ and 1 to place $2^{s-1}$ if $w_{j+1} \geq 2^{s-1}$.

Proof. The first step is to notice that the operations being applied here split according to the same rule as the operations used in the truth table recursion for $g_{r, s}^{n}$. This is true since the $m$-actions in each operation always split the same way and furthermore, when we split the table for $f_{r, s}^{n}$ all of the " $h$ " and " $\tilde{h}$ " monomials do not add any new $m$-actions. Therefore, the only $m$-actions added on during each operation split are determined by the recursion for $g_{r, s}^{n}$ and we can use Definition 7 to determine how the operations split.

We now use induction to prove that $A^{k} \cdot v$ is the column vector where the $i$ th entry is the number of times the operation $O_{i}^{n-s-k+1}$ is applied to $T_{s+k}^{j}$, or, the column vector for the $k$ th level of expansion. 
The base case occurs when $k=1$. From the remark above and the construction of $A$ it is clear that $A \cdot v$ will be a column vector where the $i$ th entry is the number of times the operation $O_{i}^{n-s}$ is applied to $T_{s+1}^{j}$.

Now by the induction hypothesis assume that $A^{k-1} \cdot v$ is the column vector for the $(k-1)$ th level of expansion. Now look at $A^{k} \cdot v$. We know that $A^{k-1} \cdot v$ is the vector which keeps track of the number of times each operation is applied to $T_{s+k-1}^{j}$. To split each portion of the truth table once more, take $A \cdot\left(A^{k-1} \cdot v\right)$ to get the column vector which keeps track of the number of times each operation is applied to $T_{s+k}^{j}$. Therefore, $A^{k} \cdot v$ is the column vector of the $k$ th level of expansion.

Finally, since $A^{k} \cdot v$ is the column vector for the $k$ th level of expansion of

$$
\begin{aligned}
& O_{w_{j}}^{n-s+1} \quad O_{w_{j+1}}^{n-s+1} \\
& T_{s}^{j} \| T_{s}^{j},
\end{aligned}
$$

to get the weight of this table when it is split into $2^{k}$ pairs, take the sum of the weight of each piece. $A^{k} \cdot v$ keeps track of the operations applied to each piece. If element $i$ of this column vector is negative, this means that we want the complement of operation $O_{i}^{n-s-k+1}$. The weight of the complement applied to $T_{s+k}^{j}$ is

$$
2^{n-s-k}-\mathrm{wt}\left(\begin{array}{c}
O_{i}^{n-s-k+1} \\
T_{s+k}^{j}
\end{array}\right) \text {. }
$$

Therefore, taking

$$
\left(\mathrm{wt}\left(\begin{array}{c}
O_{0}^{n-s-k+1} \\
T_{s+k}^{j}
\end{array}\right) \cdots \mathrm{wt}\left(\begin{array}{c}
O_{2^{s-1}-1}^{n-s-k+1} \\
T_{s+k}^{j}
\end{array}\right) 2^{n-s-k}\right) \cdot\left(A^{k} \cdot v\right)
$$

will give the weight of the table.

Theorem 3. The recursion of weights determined for $T\left(g_{r, s}^{n}\right)$ in Section 4 is also satisfied by $T\left(f_{r, s}^{n}\right)$. In particular, if $m(x)=x^{k}+d_{k-1} x^{k-1}+\cdots+d_{1} x+d_{0}$ is the minimal polynomial of the matrix A defined in Section 4, then

$$
\operatorname{wt}\left(f_{r, s}^{n}\right)=-d_{k-1} \operatorname{wt}\left(f_{r, s}^{n-1}\right)-\cdots-d_{1} \operatorname{wt}\left(f_{r, s}^{n-(k-1)}\right)-d_{0} \operatorname{wt}\left(f_{r, s}^{n-k}\right) .
$$

Proof. From Lemma 13 and Definition 12, when looking at the recursion of the truth table for $T\left(f_{r, s}^{n}\right)$, it is clear that for each pair of splits,

$$
\begin{aligned}
& O_{w_{j}}^{n-s+1} \quad O_{w_{j+1}}^{n-s+1} \\
& T_{s}^{j} \| T_{s}^{j},
\end{aligned}
$$


the recursion for weights of this portion of the truth table is induced by the polynomial $m(x)$, the minimal polynomial of $A$. In other words, when we split the table of $f_{r, s}^{n}$ into $2^{s-1}$ portions, every one of these portions satisfies the same recursion for weights defined by $m(x)$.

Now take each pair of splits and begin pairing them up, from left to right, so that the table for $T\left(f_{r, s}^{n}\right)$ is broken up into $2^{s-2}$ portions (since the truth table recursion initially splits it into $2^{s}$ portions) and we now have groups of four. In each of these groups, a recursion for weights of the first half and the second half is defined by the same polynomial. Therefore, by Lemma 12, a recursion for weights of the entire portion is defined by the polynomial $m(x)$.

Repeat this process of pairing up portions and then applying Lemma 12 until there are only two portions left. We now have that the first half of $T\left(f_{r, s}^{n}\right)$ satisfies a recursion of weights defined by $m(x)$ and the second half of $T\left(f_{r, s}^{n}\right)$ satisfies a recursion of weights defined by $m(x)$. Apply Lemma 12 one final time to get that $T\left(f_{r, s}^{n}\right)$ satisfies the recursion for weights defined by $m(x)$. The recursion defined by $m(x)$ is also the recursion we found for $T\left(g_{r, s}^{n}\right)$. Therefore, $T\left(f_{r, s}^{n}\right)$ and $T\left(g_{r, s}^{n}\right)$ satisfy the same recursion for the weights.

\section{Conclusion}

In this paper we were able to associate a recursion for the weights of cubic monomial rotation symmetric Boolean functions with a recursion for the weights of the simpler "shortened" Boolean functions $g_{r, s}^{n}$, also of degree 3. Some further work and generalization of these results could potentially prove the same result for monomial rotation symmetric Boolean functions in higher degrees.

Another interesting case to look at would be rotation symmetric Boolean functions generated by more than one monomial. If a recursion for the weights of the "shortened" versions of these functions could be determined, then we expect that the rotation symmetric Boolean functions would satisfy the same recursion for the weights.

\section{A Examples}

This appendix provides an example that can be followed while reading the paper. It demonstrates how to use the definitions, lemmas and theorems and gives an example of each conclusion found.

\section{A.1 Examples using $\boldsymbol{g}_{\boldsymbol{r}, \boldsymbol{s}}^{\boldsymbol{n}}$}

In this section we will look at the function $g_{2,4}^{n}$ for various values of $n$. 
We begin by identifying the $m$-actions for $g_{2,4}^{n}$ as defined in Definition 4 and elaborated in Lemma 2.

Example 1. $g_{2,4}^{n-1}$ has 4 possible $m$-actions: $m_{1}^{n}, m_{2}^{n}, m_{3}^{n}, m_{4}^{n}$. Using Definition 4 we can explicitly determine what the $m$-sequences for these $m$-actions will be. For each $m$-action we begin the same way and determine from Lemma 1 that $T_{n}(1,2,4)=0_{2^{n-1}} 0_{2^{n-2}}\left(0_{2^{n-4}} 1_{2^{n-4}}\right)_{2^{1}}$. We now split this sequence into $2^{4}, 2^{3}$, $2^{2}$, or $2^{1}$ equally sized portions, for each of the $m$-actions respectively:

1. $T_{n}(1,2,4)=\left(0_{2^{n-4}}\right)_{12}\left\|0_{2^{n-4}}\right\| 1_{2^{n-4}}\left\|0_{2^{n-4}}\right\| 1_{2^{n-4}}$ ，

2. $T_{n}(1,2,4)=\left(0_{2^{n-3}}\right)_{6}\left\|0_{2^{n-4}} 1_{2^{n-4}}\right\| 0_{2^{n-4}} 1_{2^{n-4}}$,

3. $T_{n}(1,2,4)=0_{2^{n-2}}\left\|0_{2^{n-2}}\right\| 0_{2^{n-2}} \|\left(0_{2^{n-4}} 1_{2^{n-4}}\right)_{2^{1}}$,

4. $\quad T_{n}(1,2,4)=0_{2^{n-1}} \| 0_{2^{n-2}}\left(0_{2^{n-4}} 1_{2^{n-4}}\right)_{2}$.

Taking the last portion only and stretching it to a length of $2^{n-1}$, we have the $m$-sequence for each action:

1. $m=1_{2^{n-1}}$,

2. $\quad m=0_{2^{n-2}} 1_{2^{n-2}}$,

3. $m=\left(0_{2^{n-3}} 1_{2^{n-3}}\right)_{2}$,

4. $\quad m=0_{2^{n-2}}\left(0_{2^{n-4}} 1_{2^{n-4}}\right)_{2}$.

A comparison shows that this result is consistent with Lemma 2.

Now that we have the $m$-actions defined, let us build a truth table recursion for $n=5$ given the table for $n=4$.

Example 2. Using direct computation, we see that the truth table for $g_{2,4}^{4}=$ $x_{1} x_{2} x_{4}$ is

$$
T\left(g_{2,4}^{4}\right)=00000000 \quad 00000101 .
$$

Now notice that $\sigma\left(g_{2,4}^{4}\right)=x_{2} x_{3} x_{5}$ and by direct computation, the truth table for $\sigma\left(g_{2,4}^{4}\right)$ in 5 variables is

$$
\begin{aligned}
T\left(\sigma\left(g_{2,4}^{4}\right)\right. & =00000000 \quad 0000010100000000 \quad 00000101 \\
& =T\left(g_{2,4}^{4}\right) \| T\left(g_{2,4}^{4}\right),
\end{aligned}
$$

as stated in Lemma 3. Furthermore, by Lemma 4, we see that the truth table for $g_{2,4}^{5}=x_{1} x_{2} x_{4}+x_{2} x_{3} x_{5}$ is

$$
\begin{gathered}
m_{4}^{5} \\
T\left(g_{2,4}^{4}\right) \| T\left(g_{2,4}^{4}\right) .
\end{gathered}
$$


From Example 1, the $m$-sequence for $m_{4}^{5}$ is

$$
0_{2^{3}}\left(0_{2^{1}} 1_{2^{1}}\right)_{2}=00000000 \quad 00110011
$$

We Xor this with $T\left(g_{2,4}^{4}\right)$ to get 0000000000110110 . Therefore, the truth table for $g_{2,4}^{5}$ is

$$
T\left(g_{2,4}^{5}\right)=00000000 \quad 00000101 \quad 00000000 \quad 00110110
$$

which can be verified by direct computation. By repeatedly doing this process we can also determine the truth table for larger values of $n$ :

$$
\begin{aligned}
& T\left(g_{2,4}^{6}\right)=00000000 \quad 00000101 \quad 00000000 \quad 00110110 \\
& \begin{array}{lllll}
00000000 & 00000101 & 00001111 & 00111001
\end{array} \\
& T\left(g_{2,4}^{7}\right)=00000000 \quad 00000101 \quad 00000000 \quad 00110110 \\
& \begin{array}{lllll}
00000000 & 00000101 & 00001111 & 00111001
\end{array} \\
& \begin{array}{llll}
00000000 & 00000101 & 00000000 & 00110110
\end{array} \\
& 00000000 \quad 11111010 \quad 00001111 \quad 11000110 .
\end{aligned}
$$

With the truth table recursion for $g_{2,4}^{n}$ identified, we can now look at a recursion for the weights. The first thing we need to identify is all of the operations and how they split.

Example 3. From Example 1, we have the list of all of the $m$-actions that can be applied to $g_{2,4}^{n}$. We would now like to determine how each of these $m$-actions split as defined in Definition 5. Looking at Lemma 5, we see that

$$
\begin{aligned}
& \text { 1. } m_{1}^{n}: \text { left side is } m_{1}^{n-1} \text { and right side is } m_{1}^{n-1} \text {, } \\
& \text { 2. } m_{2}^{n}: \text { left side is empty and right side is } m_{1}^{n-1} \text {, } \\
& \text { 3. } m_{3}^{n}: \text { left side is } m_{2}^{n-1} \text { and right side is } m_{2}^{n-1} \text {, } \\
& \text { 4. } m_{4}^{n}: \text { left side is empty and right side is } m_{3}^{n-1} \text {, }
\end{aligned}
$$

and each of these $m$-action splits can be easily verified by looking at the $m$ sequences found in Example 1. 
We can also identify the $2^{4}$ operations defined in Definition 6 .

$$
\begin{array}{rlrl}
O_{0}^{n} & =\emptyset, & & O_{8}^{n}=\left\{m_{1}^{n}\right\}, \\
O_{1}^{n} & =\left\{m_{4}^{n}\right\}, & O_{9}^{n} & =\left\{m_{1}^{n}, m_{4}^{n}\right\}, \\
O_{2}^{n} & =\left\{m_{3}^{n}\right\}, & O_{10}^{n} & =\left\{m_{1}^{n}, m_{3}^{n}\right\}, \\
O_{3}^{n} & =\left\{m_{3}^{n}, m_{4}^{n}\right\}, & O_{11}^{n}=\left\{m_{1}^{n}, m_{3}^{n}, m_{4}^{n}\right\}, \\
O_{4}^{n}=\left\{m_{2}^{n}\right\}, & O_{12}^{n}=\left\{m_{1}^{n}, m_{2}^{n}\right\}, \\
O_{5}^{n}=\left\{m_{2}^{n}, m_{4}^{n}\right\}, & O_{13}^{n}=\left\{m_{1}^{n}, m_{2}^{n}, m_{4}^{n}\right\}, \\
O_{6}^{n}=\left\{m_{2}^{n}, m_{3}^{n}\right\}, & O_{14}^{n}=\left\{m_{1}^{n}, m_{2}^{n}, m_{3}^{n}\right\}, \\
O_{7}^{n}=\left\{m_{2}^{n}, m_{3}^{n}, m_{4}^{n}\right\}, & O_{15}^{n}=\left\{m_{1}^{n}, m_{2}^{n}, m_{3}^{n}, m_{4}^{n}\right\} .
\end{array}
$$

Using the $m$-action splits we just found and Definition 7, we can determine how each of the operations split as well. Written below is the pair each operation splits into, with the left side of the operation split followed by the right side.

$$
\begin{array}{ll}
O_{0}^{n}=O_{0}^{n-1}, O_{1}^{n-1}, & O_{8}^{n}=O_{8}^{n-1}, O_{9}^{n-1}, \\
O_{1}^{n}=O_{0}^{n-1}, O_{3}^{n-1}, & O_{9}^{n}=O_{8}^{n-1}, O_{11}^{n-1}, \\
O_{2}^{n}=O_{4}^{n-1}, O_{5}^{n-1}, & O_{10}^{n}=O_{12}^{n-1}, O_{13}^{n-1}, \\
O_{3}^{n}=O_{4}^{n-1}, O_{7}^{n-1}, & O_{11}^{n}=O_{12}^{n-1}, O_{15}^{n-1}, \\
O_{4}^{n}=O_{0}^{n-1}, O_{9}^{n-1}, & O_{12}^{n}=O_{8}^{n-1}, O_{1}^{n-1}, \\
O_{5}^{n}=O_{0}^{n-1}, O_{11}^{n-1}, & O_{13}^{n}=O_{8}^{n-1}, O_{3}^{n-1}, \\
O_{6}^{n}=O_{4}^{n-1}, O_{13}^{n-1}, & O_{14}^{n}=O_{12}^{n-1}, O_{5}^{n-1}, \\
O_{7}^{n}=O_{4}^{n-1}, O_{15}^{n-1}, & O_{15}^{n}=O_{12}^{n-1}, O_{7}^{n-1} .
\end{array}
$$

Therefore, we could write the first and second levels of expansion of the truth table of $g_{2,4}^{n}$ as

$$
\begin{aligned}
& T\left(g_{2,4}^{n}\right)=T\left(\begin{array}{cc}
O_{0}^{n} & O_{1}^{n} \\
g_{2,4}^{n-1}
\end{array}\right) \| T\left(g_{2,4}^{n-1}\right),
\end{aligned}
$$

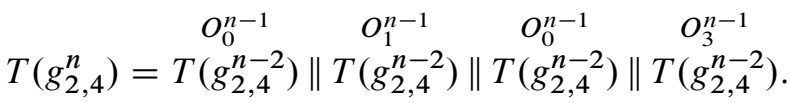

The next step in determining a recursion for the weight of $g_{2,4}^{n}$ is to find the matrix $A$. 
Example 4. $A$ is a $9 \times 9$ matrix such that the first 8 columns represent the expansion of $O_{i}^{n}$ for $0 \leq i \leq 7$. Looking at Example 3, it can be seen that the remaining 8 operations are simply the complement of the first 8 since the only difference between the two is the inclusion of $m_{1}^{n}$ in the last 8 . Therefore, looking only at the first 8 operations, we will determine the first 8 columns of $A$. This is done by adding a 1 to entry $i$ (if $0 \leq i \leq 7$ ) of column $j$ if $O_{i}^{n-1}$ is included in the splits of $O_{j}^{n}$. If $i>7$ this means that we instead want a complement, so we add a -1 into entry $i-8$ and a 1 to entry 8 . Thus, for $g_{2,4}^{n}$,

$$
A=\left[\begin{array}{ccccccccc}
1 & 1 & 0 & 0 & 1 & 1 & 0 & 0 & 0 \\
1 & 0 & 0 & 0 & -1 & 0 & 0 & 0 & 0 \\
0 & 0 & 0 & 0 & 0 & 0 & 0 & 0 & 0 \\
0 & 1 & 0 & 0 & 0 & -1 & 0 & 0 & 0 \\
0 & 0 & 1 & 1 & 0 & 0 & 1 & 1 & 0 \\
0 & 0 & 1 & 0 & 0 & 0 & -1 & 0 & 0 \\
0 & 0 & 0 & 0 & 0 & 0 & 0 & 0 & 0 \\
0 & 0 & 0 & 1 & 0 & 0 & 0 & -1 & 0 \\
0 & 0 & 0 & 0 & 1 & 1 & 1 & 1 & 2
\end{array}\right] .
$$

If we use the algorithm described in Lemma 7 to find $A$, we get the same result.

Now that we have determined the matrix $A$, we can use Lemma 8 to get

$$
\left(\operatorname { w t } \left(\begin{array}{c}
O_{0}^{n-k+1}\left(g_{2,4}^{n-k}\right) \\
\cdots
\end{array} \cdots \operatorname{wt}\left(\begin{array}{c}
O_{7}^{n-k+1}\left(T\left(g_{2,4}^{n-k}\right)\right) \\
2^{n-k}
\end{array}\right) \cdot\left(A^{k} \cdot v\right)=\operatorname{wt}\left(g_{2,4}^{n}\right),\right.\right.
$$

where $v=\left(\begin{array}{llll}1 & 0 & \cdots & 0\end{array}\right)^{T}$.

For example, when $n=7$, we can determine $\operatorname{wt}\left(g_{2,4}^{7}\right)$. Let $k=3$, then

$$
\left.\left(\begin{array}{c}
O_{0}^{5} \\
\operatorname{wt}\left(T\left(g_{2,4}^{4}\right)\right.
\end{array}\right) \cdots \operatorname{wt}\left(T\left(g_{2,4}^{5}\right)\right) \quad 2^{4}\right)=\left(\begin{array}{lllllllll}
2 & 4 & 6 & 8 & 6 & 4 & 8 & 10 & 16
\end{array}\right),
$$

where the values of the weights were determined by using the operations and $\mathrm{m}$ actions and $T\left(g_{2,4}^{4}\right)$ found in the previous examples. Finally, putting these pieces together we have

$$
\left(\begin{array}{lllllllll}
2 & 4 & 6 & 8 & 6 & 4 & 8 & 10 & 16
\end{array}\right) \cdot A^{3} \cdot v=36 .
$$

This weight can also be checked by looking at $T\left(g_{2,4}^{7}\right)$ found in Example 2 .

Finally, we have all of the information we need to determine a recursion of weights for $g_{2,4}^{n}$. 
Example 5. First notice the minimal polynomial of $A$ is $m(x)=x^{8}-2 x^{7}-2 x^{6}+$ $4 x^{5}-4 x^{3}+8 x^{2}$. We use the minimal polynomial to get

$$
\begin{aligned}
& \left(\begin{array}{cc}
O_{0}^{n-7} & O_{7}^{n-7} \\
& \mathrm{wt}\left(T\left(g_{2,4}^{n-8}\right)\right)
\end{array}\right) \cdots \mathrm{wt}\left(\begin{array}{cc}
T\left(g_{2,4}^{n-8}\right) \\
n-8
\end{array}\right) \\
& \cdot\left(A^{8}-2 A^{7}-2 A^{6}+4 A^{5}-4 A^{3}+8 A^{2}\right) \cdot v=0,
\end{aligned}
$$

where $v=\left(\begin{array}{llll}1 & 0 & \cdots & 0\end{array}\right)^{T}$. When we distribute everything through, for each term in the polynomial we get (possibly with some coefficient as well)

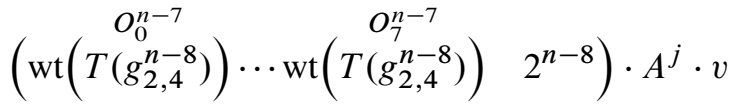

$$
\begin{aligned}
& O_{0}^{(n-8+j)-j+1} \quad O_{7}^{(n-8+j)-j+1} \\
& =\left(\operatorname{wt}\left(T\left(g_{2,4}^{(n-8+j)-j}\right)\right) \cdots \operatorname{wt}\left(T\left(g_{2,4}^{(n-8+j)-j}\right)\right) 2^{(n-8+j)-j}\right) \cdot A^{j} \cdot v \\
& =\operatorname{wt}\left(g_{2,4}^{n-8+j}\right)
\end{aligned}
$$

by Lemma 8 and where $j=8,7,6,5,3$, or 2 . Therefore, we have that $\mathrm{wt}\left(g_{2,4}^{n}\right)-2 \mathrm{wt}\left(g_{2,4}^{n-1}\right)-2 \mathrm{wt}\left(g_{2,4}^{n-2}\right)+4 \mathrm{wt}\left(g_{2,4}^{n-3}\right)-4 \mathrm{wt}\left(g_{2,4}^{n-5}\right)+8 \mathrm{wt}\left(g^{n-6}\right)=0$, or $\mathrm{wt}\left(g_{2,4}^{n}\right)=2 \mathrm{wt}\left(g_{2,4}^{n-1}\right)+2 \mathrm{wt}\left(g_{2,4}^{n-2}\right)-4 \mathrm{wt}\left(g_{2,4}^{n-3}\right)+4 \mathrm{wt}\left(g_{2,4}^{n-5}\right)-8 \mathrm{wt}\left(g^{n-6}\right)$.

\section{A.2 Examples using $f_{2,4}^{n}$}

In this section we will look at the function $f_{2,4}^{n}$ for various values of $n$. Notice from Theorem 2, that the truth table recursion for $f_{2,4}^{n}$ requires that we look at functions in $n-s$ variables. Since $s=4$ we will need to begin with a value of $n$ so that $n-s \geq 4$. Therefore, for the first few examples we will recursively build a truth table for $f_{2,4}^{8}$ from lower values of $n$. Notice that

$$
\begin{aligned}
f_{2,4}^{8}=x_{1} x_{2} x_{4}+x_{2} x_{3} x_{5}+ & x_{3} x_{4} x_{6}+x_{4} x_{5} x_{7} \\
& +x_{5} x_{6} x_{8}+x_{6} x_{7} x_{1}+x_{7} x_{8} x_{2}+x_{8} x_{1} x_{3} .
\end{aligned}
$$

We begin to look for the truth table recursion for $f_{2,4}^{8}$ by looking at the truth table recursions for some monomials in the function. Furthermore, from Theorem 2, we know that the truth table for each monomial needs to be broken up into $2^{4}$ portions. 
Example 6. From equation (9) above, it is clear that there are two monomials of the form $h_{i}^{8}: x_{6} x_{7} x_{1}$ and $x_{7} x_{8} x_{2}$.

Begin by looking at the first monomial, $h_{1}^{8}=x_{1} x_{6} x_{7}$. To break the truth table of $h_{1}^{8}$ into $2^{4}$ pieces, we will use the truth table of $h_{1}^{5}=x_{1} x_{3} x_{4}$ and equation (7). Using Lemma 9, we get $T\left(h_{1}^{5}\right)=0_{2^{4}}\left(0_{2^{2}} 0_{2} 1_{2}\right)_{2}$. Therefore, by equation (7),

$$
T\left(h_{1}^{8}\right)=\left(T_{1}^{1}\left(h_{1}^{5}\right)\right)_{2^{3}}\left\|\left(T_{1}^{2}\left(h_{1}^{5}\right)\right)_{2^{3}}=\left(0_{2^{4}}\right)_{2^{3}}\right\|\left(\left(0_{2^{2}} 0_{2} 1_{2}\right)_{2}\right)_{2^{3}} .
$$

Similarly, we can find the truth table of the second monomial, $h_{2}^{8}=x_{2} x_{7} x_{8}$, while also breaking the table up into $2^{4}$ portions. Looking at $h_{2}^{6}=x_{2} x_{5} x_{6}$, we can see that $T\left(h_{2}^{6}\right)=\left(0_{2^{4}}\left(0_{2} 01\right)_{2^{2}}\right)_{2}$ by Lemma 9 . Furthermore, using equation (7),

$$
\begin{aligned}
T\left(h_{2}^{8}\right) & =\left(T_{2}^{1}\left(h_{2}^{6}\right)\right)_{2^{2}}\left\|\left(T_{2}^{2}\left(h_{2}^{6}\right)\right)_{2^{2}}\right\|\left(T_{2}^{3}\left(h_{2}^{6}\right)\right)_{2^{2}} \|\left(T_{2}^{4}\left(h_{2}^{6}\right)\right)_{2^{2}} \\
& =\left(0_{2^{4}}\right)_{2^{2}}\left\|\left(\left(0_{2} 01\right)_{2^{2}}\right)_{2^{2}}\right\|\left(0_{2^{4}}\right)_{2^{2}} \|\left(\left(0_{2} 01\right)_{2^{2}}\right)_{2^{2}} .
\end{aligned}
$$

We now turn our attention to the monomials of the form $\tilde{h}_{i}^{8}$. From equation (9), it is clear that there is only one monomial of the form $\tilde{h}_{i}^{8}, \tilde{h}_{1}^{8}=x_{1} x_{3} x_{8}$. To find a truth table recursion for $\tilde{h}_{1}^{8}$ which breaks the table into $2^{4}$ portions we use equation (8). Therefore, we need to first find the truth table for $\tilde{h}_{1}^{7}=x_{1} x_{3} x_{7}$. Use Lemma 9 to get $T\left(\tilde{h}_{1}^{7}\right)=0_{2^{6}}\left(0_{2^{4}}(01)_{2^{3}}\right)_{2}$. Now, by equation 8 , we have

$$
T\left(\tilde{h}_{1}^{8}\right)=\left(0_{2^{4}}\right)_{2^{2}}\left\|\left(0_{2^{4}}\right)_{2}\right\|\left((01)_{2^{3}}\right)_{2}\left\|\left(0_{2^{2}}\right)_{2}\right\|\left((01)_{2^{3}}\right)_{2} .
$$

We now have all of the pieces necessary to find $T\left(f_{2,4}^{8}\right)$.

Example 7. From Example 2 we know that $T\left(g_{2,4}^{4}\right)=0000000000000101$. Furthermore, using Example 3 to determine the operations, we have

$$
\begin{array}{cccccc}
O_{0}^{5} & O_{1}^{5} & O_{0}^{5} & O_{3}^{5} & O_{0}^{5} & O_{1}^{5} \\
T\left(g_{2,4}^{8}\right)=T\left(g_{2,4}^{4}\right) \| & T\left(g_{2,4}^{4}\right) & \left\|T\left(g_{2,4}^{4}\right)\right\| & T\left(g_{2,4}^{4}\right) \| & T\left(g_{2,4}^{4}\right) \| & \left\|\left(g_{2,4}^{4}\right)\right\| \\
O_{4}^{5} & O_{7}^{5} & O_{0}^{5} & O_{1}^{5} & O_{0}^{5} & O_{3}^{5} \\
T\left(g_{2,4}^{4}\right)\left\|T\left(g_{2,4}^{4}\right)\right\| T\left(g_{2,4}^{4}\right) \| & T\left(g_{2,4}^{4}\right)\left\|T\left(g_{2,4}^{4}\right)\right\| T\left(g_{2,4}^{4}\right) \| \\
O_{0}^{5} & O_{9}^{5} & O_{4}^{5} & O_{15}^{5} & \\
T\left(g_{2,4}^{4}\right) \| & T\left(g_{2,4}^{4}\right)\left\|T\left(g_{2,4}^{4}\right)\right\| & T\left(g_{2,4}^{4}\right) .
\end{array}
$$

From Example 6 and Theorem 2, we can find the $2^{4}$ pieces of the truth table for 
$f_{2,4}^{8}$. Take the pieces and pair them up; we find each of the 8 pairs independently:
$\begin{array}{cc}O_{0}^{5} & O_{1}^{5} \\ \text { 1. } & T\left(g_{2,4}^{4}\right) \| T\left(g_{2,4}^{4}\right),\end{array}$
$\begin{array}{cc}O_{0}^{5} & O_{3}^{5} \\ \text { 2. } & T\left(g_{2,4}^{4}\right) \| T\left(g_{2,4}^{4}\right),\end{array}$

3. $\left(T\left(g_{2,4}^{4}\right) \stackrel{O_{0}^{5}}{\left.\oplus(00010001)_{2}\right)} \|\left(T\left(g_{2,4}^{4}\right) \oplus \stackrel{O_{1}^{5}}{\left.(00010001)_{2}\right),}\right.\right.$

$$
O_{4}^{5}
$$$$
O_{7}^{5}
$$

4. $\quad\left(T\left(g_{2,4}^{4}\right) \oplus(00010001)_{2}\right) \|\left(T\left(g_{2,4}^{4}\right) \oplus(00010001)_{2}\right)$,

$$
O_{0}^{5} \quad O_{1}^{5}
$$

5. $\left(T\left(g_{2,4}^{4}\right) \oplus(00000011)_{2}\right) \|\left(T\left(g_{2,4}^{4}\right) \oplus(00000011)_{2}\right)$,

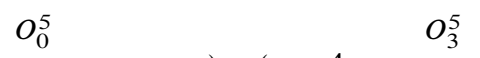

6. $\left(T\left(g_{2,4}^{4}\right) \oplus(01010110)_{2}\right) \|\left(T\left(g_{2,4}^{4}\right) \oplus(01010110)_{2}\right)$,

$$
O_{0}^{5} \quad O_{9}^{5}
$$

7. $\left(T\left(g_{2,4}^{4}\right) \oplus(00010010)_{2}\right) \|\left(T\left(g_{2,4}^{4}\right) \oplus(00010010)_{2}\right)$,

$$
O_{4}^{5}
$$

8. $\quad\left(T\left(g_{2,4}^{4}\right) \oplus(01000111)_{2}\right) \|\left(T\left(g_{2,4}^{4}\right) \oplus(01000111)_{2}\right)$.

The sequences being Xor-ed onto $T\left(g_{2,4}^{4}\right)$ in each of the lines above was found by Xor-ing together the truth tables $T\left(h_{1}^{8}\right), T\left(h_{2}^{8}\right)$, and $T\left(\tilde{h}_{1}^{8}\right)$ from Example 6 and then breaking the result into $2^{4}$ pieces. The first four pieces consist of only zeros and hence are not seen in lines 1 and 2.

By concatenating each of the lines 1-8 above we have the truth table for $f_{2,4}^{8}$. Furthermore, it is clear that each of the 8 lines consists of a pair such that the first half and second half have the same structure, as stated in Theorem 2.

Finally, we can find a recursion for the weights of $f_{2,4}^{n}$. Finding this recursion begins with looking at a recursion for each of the 8 pairs of splits, which are identified in lines $1-8$ for the case $n=8$ in the example above.

Example 8. Each portion of the table for $f_{2,4}^{n}$, defined by the 8 lines of the example above (when $n=8$ ), consists of a pair of splits that satisfies the recursion found for $g_{2,4}^{n}$ in Example 5. We will look at line 4 to demonstrate this. Using the matrix $A$ calculated in Example 4, we first show that if

$$
v=\left(\begin{array}{lllllllll}
0 & 0 & 0 & 0 & 1 & 0 & 0 & 1 & 0
\end{array}\right)^{T},
$$


then $A^{k} \cdot v$ is the $k$ th level of expansion for this portion of that table. First look at the case $k=1$. Then

$$
A \cdot v=\left(\begin{array}{lllllllll}
1 & -1 & 0 & 0 & 1 & 0 & 0 & -1 & 2
\end{array}\right)^{T} .
$$

We can see that this is the first level of expansion because if we expand line 4 once, using the operation splits identified in Example 3, we will get the four operations $O_{0}^{4}, O_{9}^{4}, O_{4}^{4}, O_{15}^{4}$. These four operations represented as a column vector is simply the vector $A \cdot v$ already found. As we continue on for greater values of $k$, it is clear that $A^{k} \cdot v$ represents the $k$ th level of expansion since the operation splits depend only on how $T\left(g_{2,4}^{n}\right)$ splits and this is what the matrix $A$ was built for.

Now, furthermore, we can find the weight of the portion defined by line 4 (for any value of $n$ such that $n-k \geq 4$ ) by noticing that

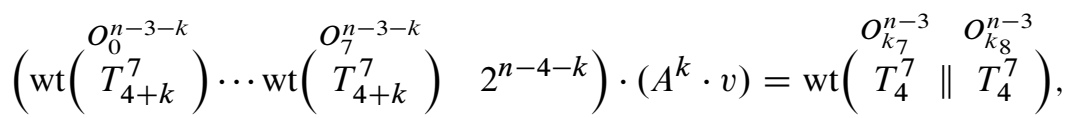

where

$$
\begin{aligned}
& O_{k_{7}}^{n-3} \quad O_{k_{8}}^{n-3} \\
& T_{4}^{7} \| T_{4}^{7}
\end{aligned}
$$

is simply line 4 , when $n=8$. Therefore, by Definition 12 , a recursion for the weight of this portion of the truth table is induced by the minimal polynomial of $A$. In a similar way, we can see that this statement is also true for all of the remaining portions of the truth table.

Now, given that each portion satisfies the recursion of weights defined by

$$
m(x)=x^{8}-2 x^{7}-2 x^{6}+4 x^{5}-4 x^{3}+8 x^{2},
$$

by Lemma 12 it must also be true that the entire truth table satisfies the recursion of weights defined by $m(x)$. Therefore, as in Example 5 for $g_{2,4}^{n}$, we have that $\mathrm{wt}\left(f_{2,4}^{n}\right)=2 \mathrm{wt}\left(f_{2,4}^{n-1}\right)+2 \mathrm{wt}\left(f_{2,4}^{n-2}\right)-4 \mathrm{wt}\left(f_{2,4}^{n-3}\right)+4 \mathrm{wt}\left(f_{2,4}^{n-5}\right)-8 \mathrm{wt}\left(f^{n-6}\right)$ and $g_{2,4}^{n}$ and $f_{2,4}^{n}$ satisfy the same recursion for the weights.

\section{Bibliography}

[1] M. L. Bileschi, T. W. Cusick and D. Padgett, Weights of Boolean cubic monomial rotation symmetric functions, Cryptogr. Commun. 4 (2012), 105-130.

[2] T. W. Cusick and P. Stănică, Fast evaluation, weights and nonlinearity of rotation symmetric functions, Discrete Math. 258 (2002), 289-301. 
[3] T. W. Cusick and P. Stănică, Cryptographic Boolean Functions and Applications, Academic Press, San Diego, 2009.

[4] H. Kim, S.-M. Park and S. G. Hahn, On the weight and nonlinearity of homogeneous rotation symmetric Boolean functions of degree 2, Discrete Appl. Math. 157 (2009), $428-432$.

[5] J. Pieprzyk and C. X. Qu, Fast hashing and rotation-symmetric functions, J. UCS 5 (1999), 20-31.

[6] X. Zhang, H. Guo, R. Feng and Y. Li, Proof of a conjecture about rotation symmetric functions, Discrete Math. 311 (2011), 1281-1289.

Received November 15, 2011; revised May 1, 2012; accepted June 13, 2012.

\section{Author information}

Alyssa Brown, Department of Mathematics, University at Buffalo, 244 Math Building, Buffalo, NY 14260, USA.

E-mail: akbrown2@buffalo.edu

Thomas W. Cusick, Department of Mathematics, University at Buffalo, 244 Math Building, Buffalo, NY 14260, USA.

E-mail: cusick@buffalo.edu 